\title{
Percutaneous osteoplasty for painful bony lesions: a technical survey
}

\author{
Won-Sung Kim and Kyung-Hoon Kim \\ Department of Anesthesia and Pain Medicine, School of Medicine, Pusan National University, Yangsan, Korea
}

Received May 4, 2021

Revised July 9, 2021

Accepted July 14, 2021

Handling Editor: Francis S. Nahm

\section{Correspondence}

Kyung-Hoon Kim

Pain Clinic, Pusan National University

Yangsan Hospital, 20 Geumo-ro,

Mulgeum-eup, Yangsan 50612, Korea

Tel: +82-55-360-1422

Fax: +82-55-360-2149

E-mail: pain@pusan.ac.kr
Percutaneous osteoplasty (POP) is defined as the injection of bone cement into various painful bony lesions, refractory to conventional therapy, as an extended technique of percutaneous vertebroplasty (PVP). POP can be applied to benign osteochondral lesions and malignant metastatic lesions throughout the whole skeleton, whereas PVP is restricted to the vertebral body. Common spinal metastases occur in the thoracic $(70 \%)$, lumbosacral $(20 \%)$, and cervical $(10 \%)$ vertebrae, in order of frequency. Extraspinal metastases into the ribs, scapulae, sternum, and humeral head commonly originate from lung and breast cancers; extraspinal metastases into the pelvis and femoral head come from prostate, urinary bladder, colon, and uterine cervical cancers. Pain is aggravated in the dependent (or weight bearing) position, or during movement (or respiration). The tenderness and imaging diagnosis should match. The supposed mechanism of pain relief in POP is the augmentation of damaged bones, thermal and chemical ablation of the nociceptive nerves, and local inhibition of tumor invasion. Adjacent (facet) joint injections may be needed prior to POP (PVP). The length and thickness of the applied needle should be chosen according to the targeted bone. Bone cement is also selected by its osteoconduction, osteoinduction, and osteogenesis. Needle route should be chosen as a shortcut to reach the target bony lesions, without damage to the nerves and vessels. POP is a promising minimally invasive procedure for immediate pain relief. This review provides a technical survey for POPs in painful bony lesions.

Key Words: Augmentation; Bone Cements; Cementoplasty; Humeral Head; Neoplasm Metastasis; Neoplasms; Osteoporosis; Pain Management; Percutaneous Osteoplasty; Ribs; Skeleton; Spinal Fractures; Sternum.

\section{INTRODUCTION}

The first percutaneous vertebroplasty (PVP) was performed in a case of hemangioma of the cervical vertebral body by Harve Deramond in France in 1984 [1]. Applications of PVP have increased from benign primary tumors and osteoporotic compression fractures to malignant osteolytic/osteoblastic/mixed painful vertebral lesions. Percutaneous osteoplasty (POP) has been developed as an extended technique of PVP for the treatment of painful bony disorders in the whole body. POPs include PVPs and extraspinal POPs (Table 1).

The human adult skeleton (206 bones) can be divided into axial (80) and appendicular (126) bones. The axial bones consist of the bones of the skull, ossicles, hyoid bone, ribs, sternum, and vertebrae. The appendicular bones are composed of the shoulder girdle, upper extremities, pelvic girdle, and lower extremities (Table 2) [2]. (a) This is an open-access article distributed under the terms of the Creative Commons Attribution Non-Commercial License (http://creativecommons.org/licenses/by-nc/4.0/), which permits unrestricted non-commercial use, distribution, and reproduction in any medium, provided the original work is properly cited.

(C) The Korean Pain Society, 2021
Author contributions: Won-Sung Kim: Investigation; Kyung-Hoon Kim: Writing/manuscript preparation. 
Table 1. Comparison between percutaneous vertebroplasty (PVP) and percutaneous osteoplasty (POP)

\begin{tabular}{|c|c|c|}
\hline Items for comparison & PVP & Extraspinal POP \\
\hline Target bone & Vertebral body & $\begin{array}{l}\text { All bones (ribs, scapulae, sternum, humeral head, } \\
\text { pelvic bones, femoral head, and talus) }\end{array}$ \\
\hline Shape of bone & Irregular bone & Flat, long, or short bones \\
\hline Incidental pain provocation & In a weight-bearing position & In a dependent position \\
\hline $\begin{array}{l}\text { Adjacent joint pain due to burden of } \\
\text { weight-bearing or immobilization }\end{array}$ & Facet joints & Glenohumeral joints, sacroiliac joints, and ankle joints \\
\hline Causative lesion & $\begin{array}{l}\text { Benign osteoporosis or primary benign tumor, } \\
\text { malignant metastatic osteolytic/osteoblastic lesions }\end{array}$ & $\begin{array}{l}\text { Benign osteochondral lesion of talus (OCT), metastatic } \\
\text { osteolytic/osteoblastic lesions }\end{array}$ \\
\hline Bone cements & Polymethylmethacrylate (PMMA) & $\begin{array}{l}\text { PMMA, calcium phosphate cements (CPCs) in OCT, } \\
\text { glass polyalkenoate cements (GPCs) }\end{array}$ \\
\hline
\end{tabular}

Table 2. Axial versus appendicular skeleton

\begin{tabular}{|c|c|c|}
\hline \multicolumn{2}{|c|}{ Bones of axial skeleton (80) } & Bones of appendicular skeleton (126) \\
\hline \multicolumn{2}{|l|}{ Skull (28) } & Should girdle (4): clavicle (2), scapula (2) \\
\hline \multicolumn{2}{|c|}{$\begin{array}{l}\text { Cranial bones (8): parietal (2), temporal (2), frontal (1), occipital (1), } \\
\text { ethmoid (1), sphenoid (1) }\end{array}$} & $\begin{array}{l}\text { Upper extremities (60): humerus (2), radius (2), ulna (2), carpals (16), } \\
\text { metacarpals (10), phalanges (28) }\end{array}$ \\
\hline \multicolumn{3}{|c|}{$\begin{array}{l}\text { Facial bones (14): maxilla (2), mandible (1), zygomatic (2), nasal (2), } \\
\text { palatine (2), inferior nasal concha (2), lacrimal (2), vomer (1) }\end{array}$} \\
\hline \multicolumn{3}{|c|}{ Auditory ossicles (6): malleus (2), incus (2), stapes (2) } \\
\hline \multicolumn{2}{|l|}{ Hyoid (1) } & Pelvic girdle (2): coxal (hip) bones (2) \\
\hline \multicolumn{2}{|c|}{$\begin{array}{l}\text { Vertebral column (26): cervical spine (7), thoracic spine (12), lumbar } \\
\text { spine (5), sacrum (1), coccyx (1) }\end{array}$} & $\begin{array}{l}\text { Lower extremities (60): femur (2), tibia (2), fibula (2), patella (2), tarsals (14), } \\
\text { metatarsals (10), phalanges (28) }\end{array}$ \\
\hline \multicolumn{3}{|c|}{ Thoracic cage (25): sternum (1), ribs (24) } \\
\hline \multicolumn{3}{|c|}{ The numbers in parentheses indicate the number of bones. } \\
\hline Shape & \multicolumn{2}{|r|}{ Bones } \\
\hline \multirow[t]{2}{*}{ Long bones } & Upper extremities & Clavicle, humerus, radius, ulna, metacarpus, phalanges \\
\hline & Lower extremities & Femur, tibia, fibula, metatarsus, phalanges \\
\hline Short bones & Carpus, tarsus & \\
\hline \multirow[t]{4}{*}{ Flat bones } & Head & Frontal bone, parietal bones, occipital bones, lacrimal bones, nasal bones, vomer \\
\hline & Shoulder girdle & Scapulae \\
\hline & Thoracic cage & Sternum, ribs \\
\hline & Pelvic girdle & Hip bones \\
\hline Irregular bones & \multicolumn{2}{|c|}{$\begin{array}{l}\text { Sphenoid bone, ethmoid bone, palatine bones, temporal bones, zygomatic bones, inferior nasal conchae, maxilla } \\
\text { bones, mandible, hyoid bone, vertebrae }\end{array}$} \\
\hline Sesamoid bones & \multicolumn{2}{|l|}{ Patella } \\
\hline
\end{tabular}

Various bones can also be classified by their shapes: long, short, flat, sesamoid, and irregular bones (Table 3). In POP, the classification by the shape of bones is important when inserting the needle and placing the bone cement, as well as in preserving the effectiveness of the cement augmentation against weight bearing in a dependent position. For example, POP in the humeral head is enough to improve its strength, however, POP in the femoral head needs additional intramedullary nailing in the femoral shaft to bear the weight of the body.

Bone is the third most frequent site of metastasis, after the lung and liver. Prostate and breast cancers are responsible in the majority of bone metastases (up to 70\%), followed by thyroid, lung, urinary bladder, and renal cancers, and finally melanoma. Median survival rate after diagnosis of bone metastases is known to be 4 years in thyroid cancer, 2 years in breast cancer, 1-4.5 years in prostate cancer, 1 year in renal cancer, 6-9 months in urinary bladder cancer, and 6 months in lung cancer and melanoma. Fortunately, the 3 most common cancers which develop bone metastases (prostate, breast, and thyroid cancers) show relatively high median survival rates [3]. 
The primary tumors in the neck and thorax, such as those of the thyroid gland, breast, and lung, frequently metastasize to the cervicothoracic vertebrae, ribs, scapulae, sternum, and humeral head; the primary tumors of the abdomen and pelvis, such as those of the stomach, colon, prostate, urinary system, and uterine cervix, invade the lumbosacral vertebrae, pelvic bones (the ilium, ischium, and pubis), and femoral head.

This technical survey introduces various POPs in axial/ appendicular or malignant/benign painful bony lesions.

\section{MAIN BODY}

\section{General concepts}

1) Mechanisms of pain relief in POP, including PVP

The exact mechanisms of pain relief in PVP are not fully understood. POP creates 1) mechanical stabilization of microfractures, resulting in a reduction of irritation to free nerve endings and restoring strength and stiffness in the vertebral body, 2) thermal ablation, as high as $70^{\circ} \mathrm{C}$, to the free nerve endings, 3) decompression of intraosseous pressure following compression fractures in the vertebral body, and 4) an antitumor effect due to the local toxicity of polymethylmethacrylate (PMMA). The mechanisms of pain relief in POP for various shapes of bones do not seem different from those of PVP [4].

2) Basic principle for needle insertion and selecting needle gauge sizes

The most important concern for inserting the needle in POP is choosing the shortcut from the skin to targeted bone lesions, in order to reduce procedural time and effort while avoiding damage to the adjacent or traversing nerves and vessels of the targeted bone. Vulnerable vessels and nerves of the targeted bones will be discussed in each POP.

The best method for reducing the risk of piercing important nerves and vessels is not only having knowledge about anatomy but also reducing the speed of needle insertion near these structures in order to give them enough time to escape from the approaching needle.

According to the thickness of the targeted bone or the approaching route of the needle, selection of the needle gauge size and length should be different. An 11 gauge, $10 \mathrm{~cm}$ long needle is chosen for the vertebral bodies from the typical cervical to the sacral vertebrae, humeral head, scapula, femoral head, greater or lesser trochanter of the femur, as well as the pelvic bones, including the ilium, is- chium, and pubic bones. A 13 gauge, $10 \mathrm{~cm}$ long needle is selected for the lateral masses of the atlas and the body of the axis. A 13 gauge, $5 \mathrm{~cm}$ long needle is recommended for the ribs and sternal body.

\section{3) Bone cement}

It should be considered whether the inserted cement helps improve strength and stability in a certain bone, such as long, short, flat, or irregular bones. It is better to discuss this in each POP case, according to the shape of the bones. The result for insertion of the bone cement into the head or shaft (with a medullary cavity) in long bones (humerus and femur) or flat bones (ribs, sternum, scapulae, or ilium) may be quite different in regards to improving strength and stability. The flat bones are composed of 2 outer layers of compact (trabecular) bone and intervening cancellous (spongy) bone. The long bones consist of proximal and distal epiphyses connected to the diaphysis via the metaphysis.

Commonly available injectable bone cements (IBCs) include (1) PMMA or acrylic bone cements (ABCs), (2) calcium phosphate cements (CPCs), calcium sulfate cements (CSCs), or hydroxyapatite cements (HACs: tetra-calcium phosphate and di-calcium phosphate anhydrous powders), and (3) glass polyalkenoate (ionomer) cements (GPCs or GICs, alumino-silicate glass) [5-9].

Bone cements are selected according to their osteoconduction, osteoinduction, and osteogenesis [9]. CPCs or HACs, instead of PMMA, have been used for the treatment of benign osteochondral lesions (OCLs) of the talus or tibia for osteoconduction, osteoinduction, and osteogenesis [10].

\section{4) Necessity of adjacent (facet) joint injections prior to POP (PVP)}

Facet joint injections or medial branch blocks are required when the vertebral body (anterior component of the spine) cannot bear the weight due to benign osteoporotic compression fracture or malignant metastatic osteolytic compression fracture, resulting in overloaded facet joints (posterior components of the spine). Unilateral or bilateral facet joint injections are needed prior to the PVP because: (1) they help physicians confirm the exact currently painful vertebrae among mixed old and new compressed vertebrae, (2) patients can cooperate in maintaining a prone position during the PVP under local anesthesia or monitored anesthetic care, and (3) it can reduce patients' dissatisfaction from incomplete pain relief from PVP while facet joint pain remains $[11,12]$.

Glenohumeral joint injection is commonly required in a 
painful humeral head metastasis, resulting in a long-term immobilization of the glenohumeral or acromioclavicular joint due to the painful bony lesion. Sacroiliac joint injections are also needed in painful sacral or iliac metastatic fractures due to the joint being overloaded, which produces Honda sign in bone scans.

5) Deciding whether to perform POP according to life expectancy of patients with bone metastases and evaluation for effectiveness of POP

The Mirels score is helpful in assessing the risk of pathologic fracture due to lytic lesions of the long bones, based on these variables with 3 grades, including the site of the pathologic fracture, its size, pain intensity, and the presence of a lytic lesion. If the total score is greater than 9, the need for prophylactic fixation, due to a high risk of fracture, should be considered [13].

The revised Tokuhashi score is useful for assessing the life expectancy of patients with bone metastases. The score includes 6 variables, including general condition based on performance status, the number of extraspinal bone metastases foci, number of metastases in the vertebral body, metastases to the major internal organs, primary site of the cancer, and palsy, according to the Frankel classification. Life expectancy for proper treatment is supposed to be less than 6 months for conservative treatment, 6-12 months for palliative surgery, or 12 months or more for excisional surgery, these periods being determined by a total score of less than 8, 9-11, or 12-15, respectively [14]. Even though POPs are considered palliative surgery, they can be performed on any patient who can tolerate a bone biopsy.

The Karnofsky performance scale (KPS) is useful for comparing the effectiveness of therapies, including POPs, and for assessing the prognosis of patients. The lower the KPS score, the worse the chance of survival [15]. The Eastern Cooperative Oncology Group performance status (ECOG PS) is also commonly used for evaluation for physical performance status [16]. The higher the ECOG PS score, the worse the chance of survival. These evaluation systems use a markedly different score for the same performance status (Table 4). These PS evaluation systems can be used for evaluating the effectiveness of POP before and after the procedure.

\section{6) Diagnostic procedures for painful metastatic bony lesions}

It is common to find painful metastatic bony lesions after the diagnosis of primary malignant lesions. However, it is not rare to find painful metastatic bony lesions before the diagnosis of the primary malignant lesions or lesions of an unknown origin.

Most patients complain of aggravation from incidental pain in a certain position or during positional changes. Tenderness on the bony lesions can suggest the presence of fracture. For a confirmation of the presence of metastatic bony lesions through history and physical examination, imaging diagnosis is required, such as X-ray, computed tomography or positron emission tomography-computed tomography/magnetic resonance imaging, and bone scans.

\section{Percutaneous vertebroplasty (spinal POP)}

Metastases to the axial skeleton, rather than the appendicular skeleton, are common due to its hematopoietically active red bone marrow, paravertebral network, and micro-environment support, based on the seed-and-soil hypothesis [3].

1) Cervical vertebrae

(1) Atypical cervical vertebrae

\section{(1) $\mathrm{Cl}$ vertebra}

The atlas has no vertebral body. Instead of a vertebral body, the lateral masses, which are the bulkiest and hardest parts of the atlas, support the weight of the head. Each mass has the superior and inferior articular processes. The superior articular process has a concave articular surface corresponding with the occipital condyle, making the atlanto-occipital (AO) joint, which allows the nodding (yes) movement of the head. The inferior articular process has a flat or convex articular surface corresponding with the superior articular process of the axis, making the atlantoaxial (AA) joint, which permits rotational (no) movement of the head [17].

The lateral masses, the major weight bearing units of the atlas, become the targeted metastatic bony structures for osteoplasty. Candidates for POP at the lateral mass of the atlas are not able to bear the weight of the head due to the osteolytic metastatic lesion of the lateral mass. They usually complain of intractable pain in the suboccipital area with a limited range of neck motion. They can only sleep on the side opposite the lesion with a pillow.

On physical examination, there is tenderness in the one side of the suboccipital area and severe limitation of ipsilateral rotation of the neck. A destructive lesion should be found in the lateral mass of the atlas from the computed tomography/magnetic resonance imaging and bone scans (Fig. 1A).

For performing POP at the lateral mass, the patients are 


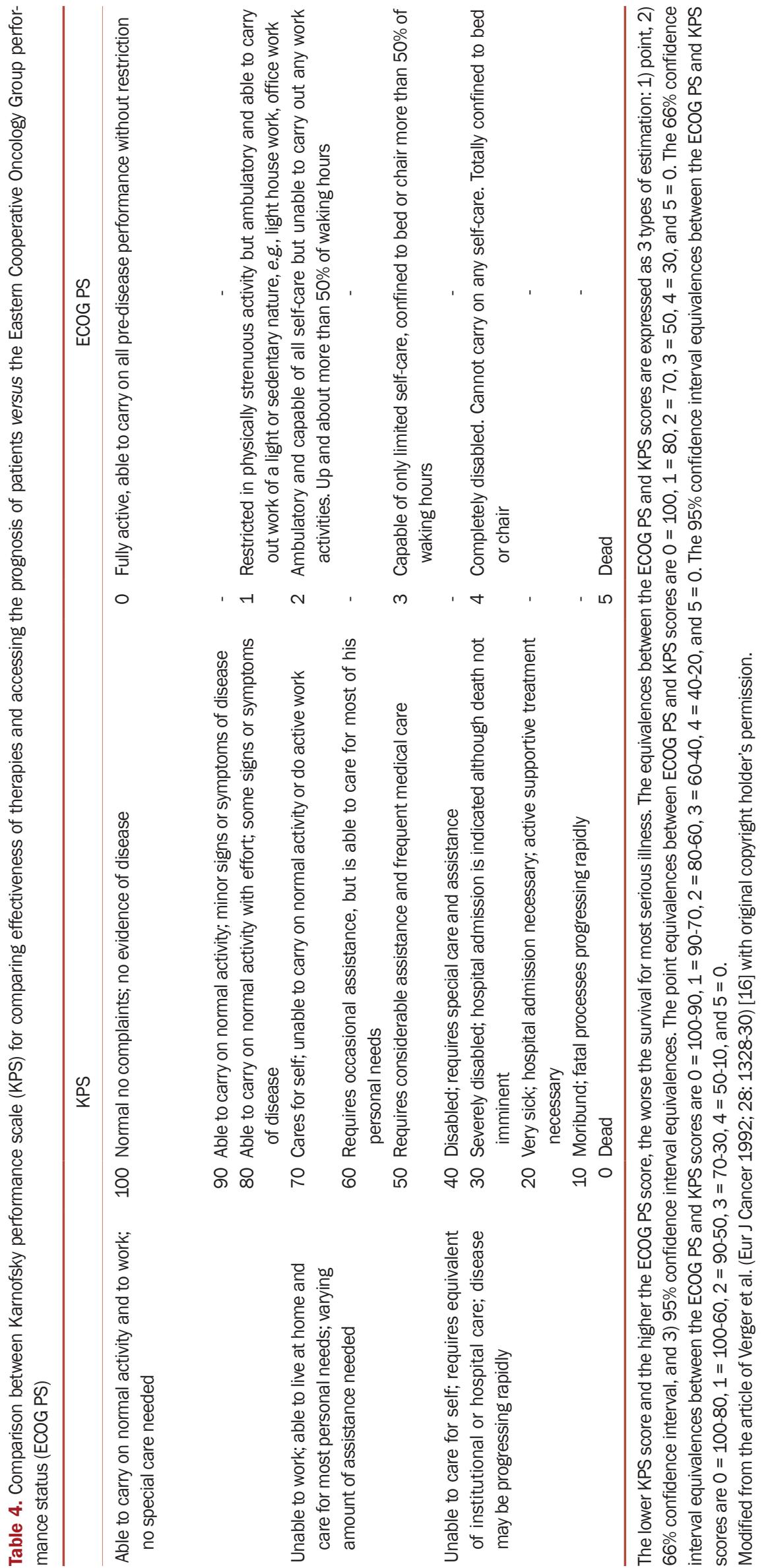


placed in the supine position with a pillow under their shoulders to create neck extension. After sterile draping, local anesthetic with $1 \%$ lidocaine is given at the point of the anticipated needle entry. A 13 gauge vertebral needle is introduced after determining the location of the carotid artery under ultrasound guidance. While advancing the needle, great care should be given to avoid piercing the vertebral artery and the spinal nerves by observing the depth of penetration using lateral fluoroscopy. After confirming the needle position in the targeted lateral mass, contrast medium is injected to observe any possible leakage of bone cement into the vessel or epidural space. The amount of bone cement for this procedure is less than 1 mL (Fig. 1B).

Immediately after POP at the lateral mass of the axis (Fig. 1C), suboccipital headache disappears and the limitation of the neck motion is decreased. The anterolateral approach for POP, under the guidance of both ultrasound and fluoroscope, is safe under local anesthesia. The transoral approach has also been introduced; however, it can be performed under general anesthesia with nasotracheal intubation [18].

AO and/or AA joint injections may be needed before/ after POPs in the $\mathrm{C} 1$ and $\mathrm{C} 2$ vertebrae, as with other facet or glenohumeral joint injections in spinal or extraspinal POPs $[11,12]$.
(2) $\mathbf{C} 2$ vertebra

The axis is composed of the body, dens, transverse processes, pedicles, pars interarticularis, and superior/inferior processes. The anteriorly located dens (odontoid process) is a characteristic structure which is connected to the body. The superior articular facets and dens of the axis (C2) articulate with the inferior articular facets and the facet for dens of the atlas (C1), respectively. The inferior articular facets of the axis articulate with the superior articular facets of the C3 vertebra.

All transverse processes in the first 6 cervical vertebrae have a pair of distinct transverse foramina, each of which contains a vertebral artery and vein. The 7 th vertebra also has a pair of transverse foramina, each of which contains only the vertebral vein, not the artery [19].

Patients with a C2 body and/or dens fracture complain of suboccipital headache and cannot sleep lying down on their back or dependent side, and aggravate their pain in an upright position. Therefore, patients can usually be found in a sitting position supporting their head with neck flexed.

Plain X-ray, computed tomography/magnetic resonance imaging, and bone scans should match with the above symptoms and signs (Fig. 2A). Patients are placed in the supine position with neck slightly extended. The entry point of the puncture for the anterolateral approach is the edge of the mandibular angle. Ultrasound is also helpful
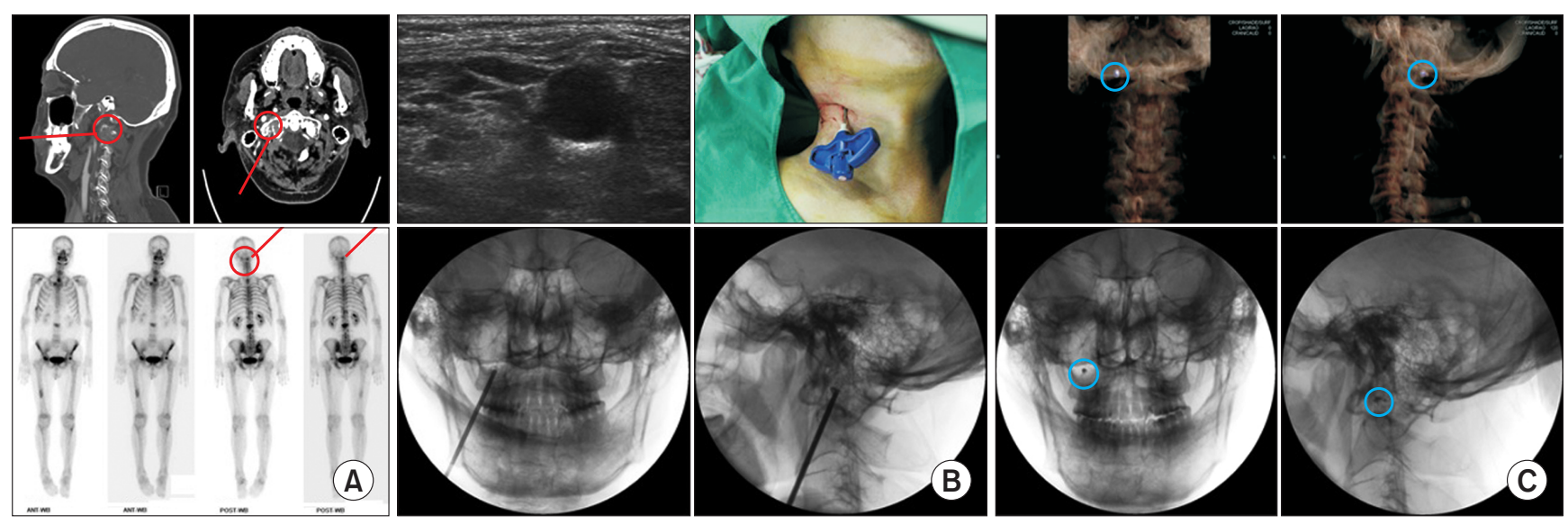

Fig. 1. Percutaneous osteoplasty in the right lateral mass of the $\mathrm{C} 1$ vertebra (atlas). (A) Preoperative computed tomography and bone scans of a metastatic destructive lesion of the right lateral mass of the atlas. The red circle indicates a destructive bony metastatic lesion in the right lateral mass of the atlas from sagittal and axial views. The bone scans show an active lesion (the red circle) on the right lateral mass in the posterior view. The characteristic feature of the anterior view is the head tilted left, to the side opposite the lesion. (B) Intraoperative ultrasonic, exterior, and fluoroscopic views of the percutaneous osteoplasty for a metastatic destructive lesion of the right lateral mass of the atlas. (Upper left) Great caution should be given to the adjacent vulnerable anatomic structures, including the carotid artery, vertebral artery, and spinal nerves. (Upper right) A 13 gauge, $10 \mathrm{~cm}$ long vertebral needle is placed in the right lateral mass. (Lower left) The anterior posterior fluoroscopic view shows a vertebral needle, placed in the lateral mass. (Lower right) The lateral fluoroscopic view shows contrast medium without leakage into the vessels or epidural space. (C) Postoperative computed tomography and fluoroscopic views after percutaneous osteoplasty for a metastatic destructive lesion of the right lateral mass of the atlas. (Upper left and right) The purplish-white dot (within a blue circle) is located in the right lateral mass in the coronal and sagittal views. (Lower left and right) The black dot within a blue circle is located in the lateral mass in the anterior posterior view and lateral fluoroscopic views. 


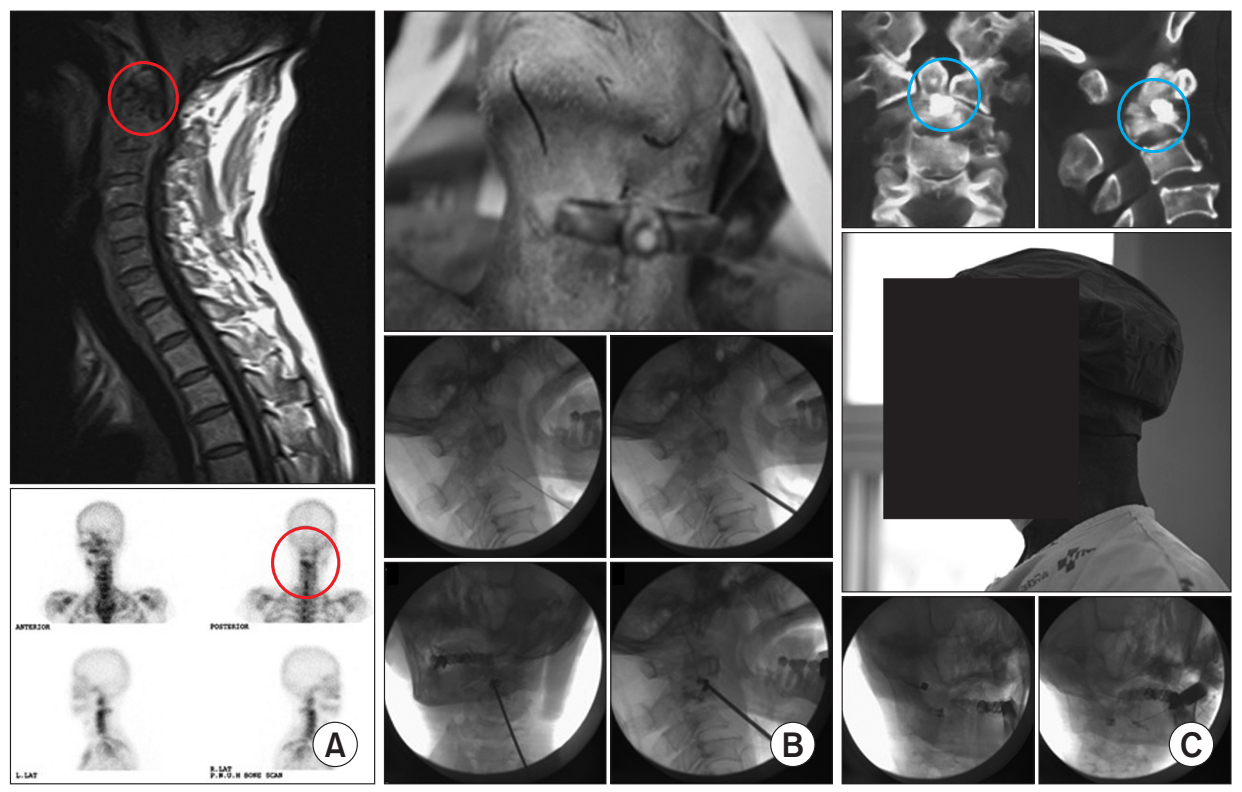

Fig. 2. Percutaneous vertebroplasty at the $\mathrm{C} 2$ body and dens. (A) Preoperative magnetic resonance imaging shows a destructive lesion (the red circle), and bone scans also show an active bone lesion (the red circle) in the $\mathrm{C} 2$ body and dens. (B) Intraoperative percutaneous vertebroplasty at the $\mathrm{C} 2$ body and dens is performed using a 13 gauge, $10 \mathrm{~cm}$ vertebral needle along the 23 gauge, $10 \mathrm{~cm}$ spinal needle for local anesthetic infiltration. (C) (Upper) Postoperative computed tomography shows a proper filling of the destructive lesion with bone cement (the blue circle). (Middle) Patients can experience immediate pain relief and partially recover their necks' range of motion. (Lower) Postor pre-operative atlanto-occipital and atlanto-axial joint injection are commonly performed.

to avoid the major vessels/nerves and tracheoesophageal complex (TEC), as with performing POP at $\mathrm{Cl}$. Along with a $10 \mathrm{~cm}, 23$ gauge spinal needle for local anesthesia from the skin to the vertebral body, a $10 \mathrm{~cm}, 13$ gauge vertebral needle is advanced and anchored to the targeted vertebral body under alternating anteroposterior and lateral fluoroscopic guidance. After removal of the stylet of the needle, contrast medium is injected to confirm any leakage into the vessels or epidural space. Bone cement, PMMA, with barium powder for increasing opacity, less than $1 \mathrm{~mL}$, is injected under continuous fluoroscopic control. Postoperative computed tomography should show proper filling with bone cement in the destructive lesion of the body and/or dens (Fig. 2B). Patients can get immediate pain relief and recovery of the range of motion of the neck. AA and AO joint injections may be needed, before or after PVP at C2 (Fig. 2C) [12].

\section{(2) Typical cervical vertebrae (C3-C7)}

Characteristic preoperative symptoms and signs are weight-bearing pain and limitation of the range of motion in the neck. The preoperative imaging diagnostic tools, including plain X-ray, bone scans, and magnetic resonance imaging/computed tomography, should be correlated with the painful metastatic lesion (Fig. 3A). Radiation therapy is somewhat helpful in relieving pain, however, the role of augmentation by PVP may be necessary in most cases.

The key principle using an anterolateral approach to the vertebral body, from the $\mathrm{C} 3$ to $\mathrm{C}$, is to avoid the TEC, internal carotid artery, and internal jugular vein. The method for pushing the TEC away from the approaching needle is the same as in performing percutaneous endoscopic cervical discectomy [20].

Right-handed operators should stand to the left side of the patient. After the left middle and index fingers split the TEC medially and carotid artery laterally, infiltration of local anesthetic, using a $10 \mathrm{~cm}, 25$ gauge spinal needle into the skin, subcutaneous tissue, and anteromedial vertebral body, is performed. While leaving the spinal needle in place, and placing the fingers with the middle finger against the TEC and the index finger against the carotid artery, a $10 \mathrm{~cm}, 11$ gauge vertebral needle is inserted alongside the spinal needle.

After anchoring the vertebral needle to the left anteromedial vertebral body by hammering against the slippery anterior longitudinal ligament, the position of the needle can be directed to the targeted bony lesion. The anterior segment of the cervical spine, the vertebral body, is a weight-bearing structure supporting the head. If the targeted bony lesion affects the anterior vertebral body, the needle can be placed into the anterior $1 / 3$ to $1 / 2$ of the vertebral body (Fig. 3B).

Even if there is a disruption of the posterior wall of the vertebral body, it is better to place the needle in the anterior $1 / 3$ of the vertebral body. The next step is to check for leakage of the contrast medium into the blood vessels or anterior epidural space. If the contrast medium is contained inside the vertebral body for a moment before leaking into the blood vessels or anterior epidural space, rather than using the usual toothpaste consistency, thicker bone cement is required. It is better, in cases using a reduced volume of injected bone cement, to rely on pain relief from thermal and chemical ablation of the nerves rather than augmentation by the injected volume. Normally, the injected cement volume is less than $2 \mathrm{~mL}$. 


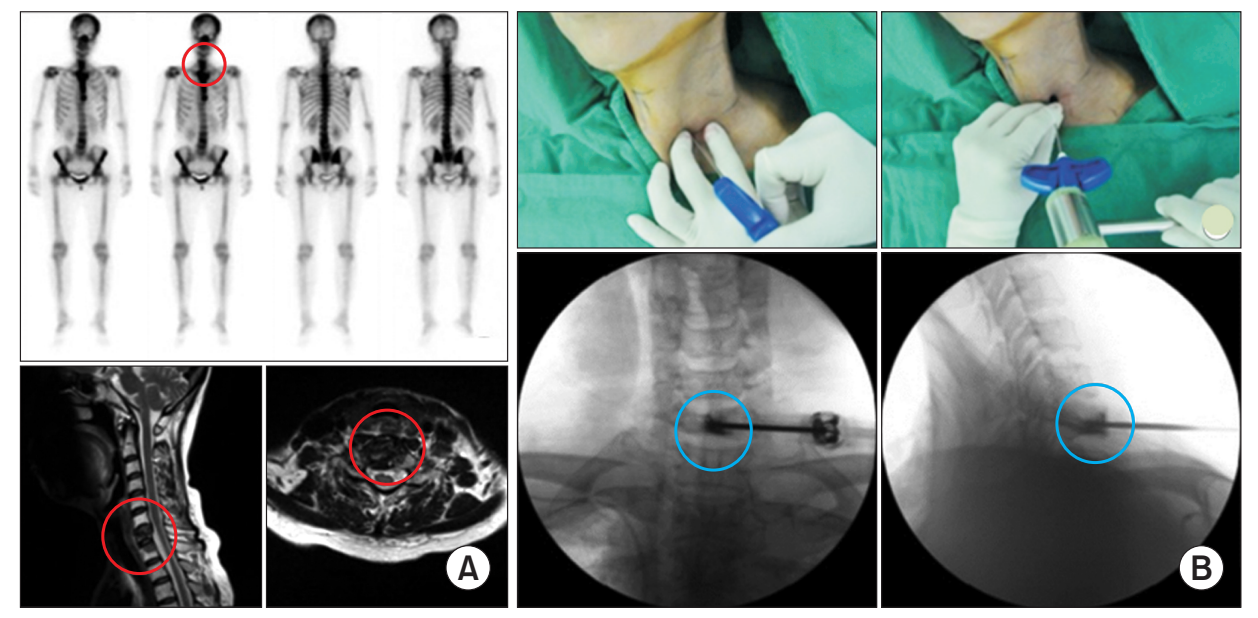

Fig. 3. Percutaneous vertebroplasty at the $\mathrm{C} 7$ body. (A) Preoperative images: Bone scans shows an active lesion (the red circle) at the $\mathrm{C} 7$ vertebral body. Magnetic resonance imaging shows a destructive lesion (the red circle) at the $\mathrm{C} 7$ vertebral body. (B) Intraoperative images. (Upper left) After pushing the tracheoesophageal complex with the middle finger and palpating the internal carotid artery with the index finger, an 11 gauge, $10 \mathrm{~cm}$ vertebral needle is inserted and advanced into the anterior $1 / 3$ of the vertebral body. (Upper right) Hammering is useful for anchoring the vertebral needle into the vertebral body through the slippery anterior longitudinal ligament. (Lower left) After confirmation of no leakage into the vessels or epidural space, bone cement (the blue circle) should be inserted into the destructive lesion. (Lower right) The target point of the needle is the anterior $1 / 3$ of the vertebral body. Adapted from the article of Seo et al. (Korean J Anesthesiol 2013; 64: 276-9) [20].

Facet joint injections may be also needed before and after PVP for the classic cervical vertebrae from C3 to C7 $[21,22]$.

\section{2) Thoracic and lumbar vertebrae}

(1) Transpedicular versus extrapedicular approach

Depending on the route of the vertebral needle passing the pedicle, the method of approach to the vertebral body can be divided into a transpedicular and extrapedicular approach. The transpedicular approach is a conventional, safe method, even though it is difficult for the needle to penetrate the hard consistency of the pedicle. It has a risk for spinal cord or dorsal root ganglion injury. The transpedicular approach can be divided into unipedicular and bipedicular methods. The unipedicular approach can be performed in the thoracolumbar vertebrae. It can reduce time, risk, and effort in performing PVP (Fig. 4) [23,24].

\section{(1) Unipedicular approach}

Patients are placed in a prone position. Monitored anesthetic care, using $30 \mathrm{mg}$ of ketorolac and $50 \mu \mathrm{g}$ of fentanyl, is performed. Facet joint injections are performed prior to PVP, if necessary. The targeted vertebral body, the midline passing through the spinous processes, the pedicle with its quadrants, especially the outer, upper paadrant, aswell as lines marking 1,2 , and 3 pedicular distances parallel to the midline should be drawn in the anteroposterior fluoroscopic view. A Kirschner (K) wire is placed as it passes from the upper pedicle to the anterior $1 / 3$ to $1 / 4$ of the targeted body in the lateral fluoroscopic view.

Local anesthesia is performed using a $10 \mathrm{~cm}, 23$ gauge spinal needle. The needle is inserted from 2-3 pedicular distances into the outer, upper pedicle in the anteroposterior fluoroscopic view after local infiltration of $1 \%$ lidocaine into the skin. A $10 \mathrm{~cm}, 11$ gauge vertebral needle is inserted along with the spinal needle after skin incision using a No. 15 blade scalpel along with kin crease. A vertebral needle is anchored to the outer, upper quadrant of the pedicle by hammering. While passing the pedicle, hammering is not recommended to avoid spinal cord or nerve root injury, and aiming of the vertebral needle upward and outward is also helpful to reduce the risk of nerve injury.

After penetrating the posterior wall of the vertebral body, rotation of the bevel of the vertebral needle toward the medial, lateral, superior, or inferior direction is helpful to reach to targeted anterior $1 / 3$ or $1 / 4$ of the vertebral body at in the midline. When the vertebral needle is placed in the vertebral body, hammering is allowed while the depth of the needle is fixed with curved Kelly forceps to prevent passing the targeted anterior $1 / 3$ to $1 / 4$ of the vertebral body. A leakage test into the epidural space or blood vessel, using contrast medium, is essential. If found, a slice of gelatin sponge is helpful and the test should be performed again [23]. The injected bone cement volume is less than 3 or $5 \mathrm{~mL}$ in the thoracic or lumbar vertebrae, respectively.

\section{(2) Extrapedicular approach}

The extrapedicular approach is selected when a pedicle 


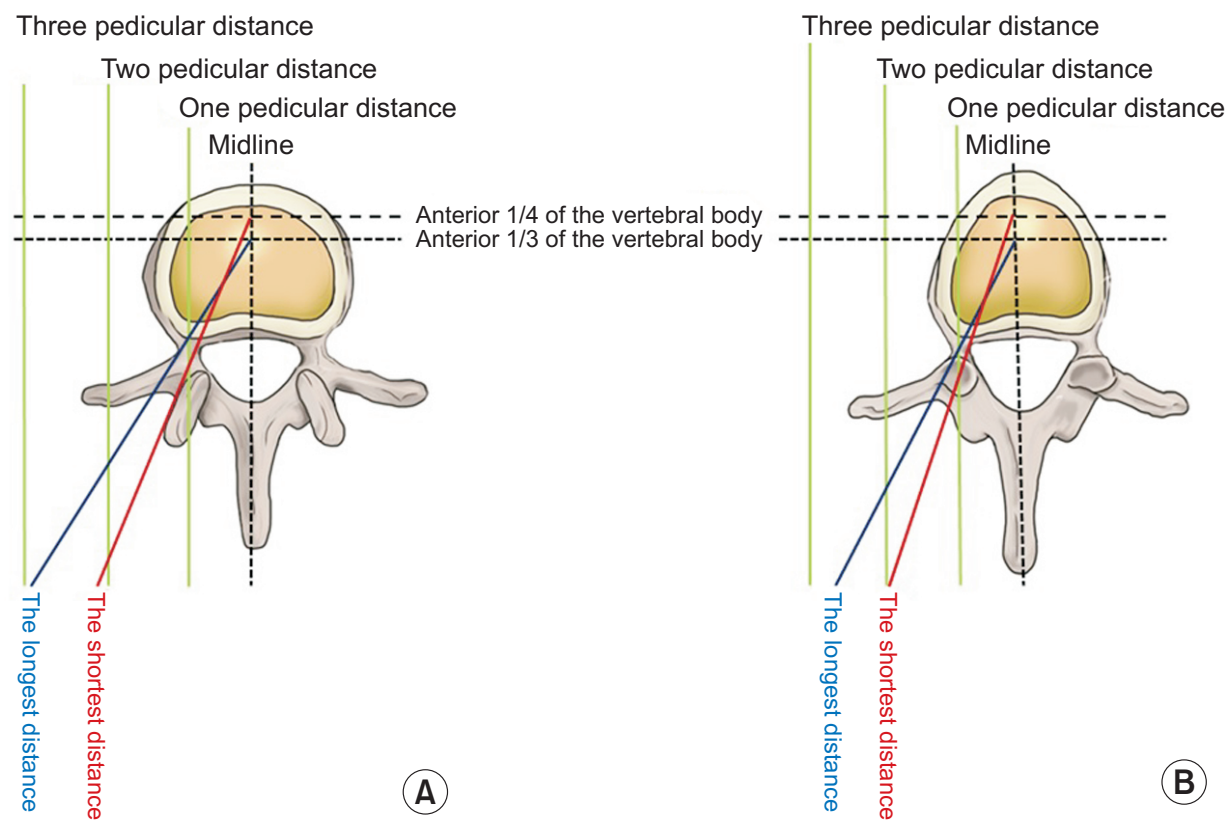

Fig. 4. Imaginary lines for the unipedicular approach in percutaneous vertebroplasties (PVPs) of the lumbar and thoracic vertebrae. If a vertebral needle passes through the structure of the pedicle, the approach method should be called "a transpedicular approach" for PVP. If not, it should be called an "extrapedicular approach". The vertebral needle should not penetrate the inner wall of the pedicle, so as not to pierce the spinal cord or dorsal root ganglion. The target point of the vertebral needle is the anterior $1 / 3$ to $1 / 4$ of the vertebral body. (A) In the lumbar vertebrae, the shortest distance from the midline is 2 pedicular distances, and the longest distance from the midline is 3 pedicular distances. (B) On the contrary, in the thoracic vertebrae, the shortest distance from the midline is 2 pedicular distances and the longest distance from the midline is 2.5 pedicular distances.

screw has already been fixed. However, the risk of piercing of the segmental arteries of the spine (posterior intercostal [T3-T11], subcostal [T12], and lumbar [L1-L4] arteries) may be increased. The posterior intercostal arteries arise directly from the thoracic aorta (T3-T11) or subclavian artery (T1-T2). The posterior intercostal artery has a dorsal and a collateral branch. The dorsal branch is divided into the spinal branch (to the spinal cord) and medial and lateral cutaneous branch (to the posterior thoracic wall). The collateral branch supplies blood to the lateral thoracic wall. The 1st to 6th anterior intercostal arteries arise directly from the internal thoracic artery, and the 7th to 9th anterior intercostal arteries indirectly from the musculophrenic artery (a branch of the internal thoracic artery), and connects to the posterior intercostal artery. The anterior intercostal artery is not present between the 10th and 11th intercostal spaces. Therefore, the posterior intercostal arteries supply each space solely. The 12th subcostal artery, below the 12th rib, arises directly from the thoracic aorta. The 4 pairs of lumbar arteries are located parallel to the intercostal arteries and subcostal artery [25-28].

The segmental arteries usually run along thoracolumbar vertebral bodies at the infra-pedicular level. Therefore, it is recommended to anchor the vertebral needle at the pedicular level and the midline of the lateral vertebral body with a reduced inserting speed. As with the transpedicular approach, the vertebral needle should target the anterior
$1 / 3$ to $1 / 4$ of the vertebral body.

(2) Benign versus malignant painful thoracolumbar lesions

It is common to predict metastatic vertebral fractures in patients with known malignancies. However, intractable back pain in a weight bearing position may be the first symptom to suggest metastatic lesions. Attention should be given in patients who are young, in a low risk group for osteoporosis, and men.

Palpation after adjacent facet joint injections among multiple metastatic vertebral fractures makes the current painful fracture levels clear. After removal of facet joint pain, it is easy to recognize precise supraspinous tenderness among multiple fractures. In addition, the effects of facet joint injections help patients to lie on their abdomen with less pain and increases cooperation during the PVP procedure under local anesthesia [11,29].

Spinal metastases, in order of frequency, are to the thoracic $(70 \%)$, lumbosacral $(20 \%)$, and cervical $(10 \%)$ vertebrae. Multiple spinal metastases range from $17 \%$ to $30 \%$. The first metastatic involvement within the vertebra is the posterior portion of the vertebral body before the pedicles. Absence of the pedicle due to metastatic destruction, in plain X-ray film, is a characteristic feature comparable to an osteoporotic compression fracture (Fig. 5) [30,31]. 
Osteoporotic vertebral compression fractures are also frequent in the thoracolumbar vertebrae [32].

\section{3) Sacrum}

The sacrum, 5 fused sacral vertebrae, is composed of the vertebral body (from the promontory to the apex of the sacrum) and alae (wings). Honda sign is a characteristic $\mathrm{H}$ shaped (wider upper and narrow lower $\mathrm{H}$ letter) increase in radioactive uptake in the sacral body and both alae, parallel to the sacroiliac joints, observed on bone scans, indicating a bilateral sacral insufficiency fracture [33].

\section{(1) Sacral body}

Sacroplasty in the sacral body needs bipedicular approaches because it is dangerous for the vertebral needle to penetrate to the midline of the sacrum (Fig. 6A). An epidurogram, usually through the S1 foramen, is helpful in avoiding neural passage prior to vertebral needle insertion into the sacral body. The amount of injected bone cement is less than $5 \mathrm{~mL}$ for each approach.

\section{(2) Sacral wings (alae)}

Sacroplasty, in each sacral wing, from the S1 to S5, can be performed by insertion of the vertebral needle once from the S1 to S5 alae, and the cement can be inserted while withdrawing the needle from the S5 to S1 alae (Fig. 6B). The sacroplasty in the ala is quite similar to performing POP in the ilium, in that caution should be given not to pierce the cauda equina or spinal nerves, or inject bone cement into the sacroiliac joints. The amount of injected bone cement is less than $10 \mathrm{~mL}$ for each sacral sacral wing. Sacroiliac joint injections with contrast medium or a steroid may be needed in performing sacroplasty of the sacral wings for both preventing leakage into the sacroiliac joint and relieving joint pain, respectively.

\section{Extraspinal POP in malignant metastases}

There are 33 irregular bones in the human body: the spine
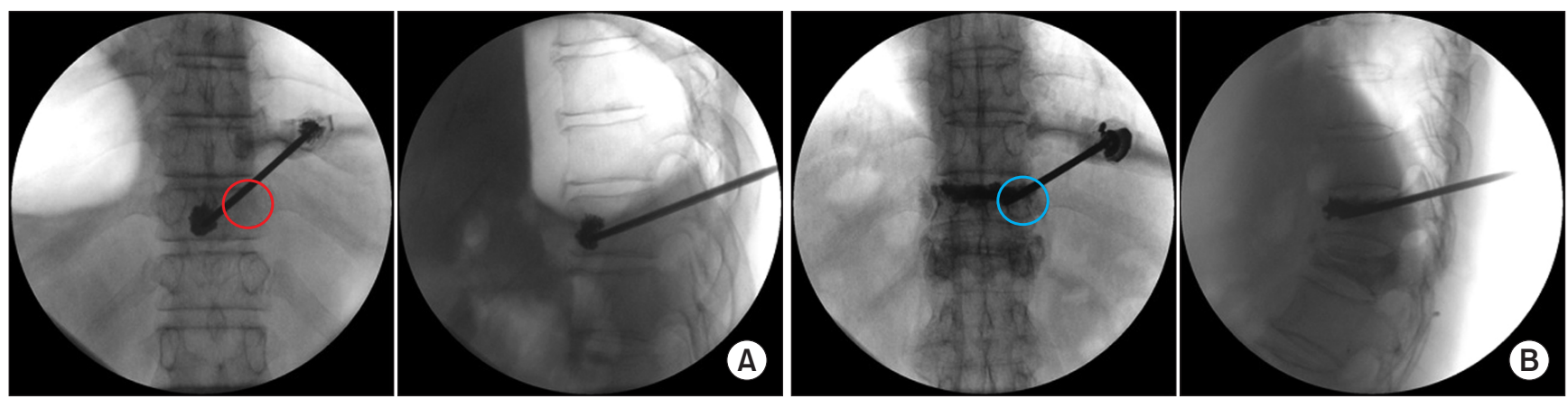

Fig. 5. Percutaneous vertebroplasty for painful osteolytic vertebral compression fracture versus osteoporotic vertebral compression fracture at T11. The pedicle of the spine is strong enough to endure osteoporosis, however, it (and the posterior vertebral body) is vulnerable to spine metastases. (A) The anteroposterior fluoroscopic view shows no right pedicle (the red circle) in the metastatic vertebral osteolytic fracture, however, (B) there is an apparent right pedicle (the blue circle) in the osteoporotic vertebral compression fracture in the anteroposterior fluoroscopic view.
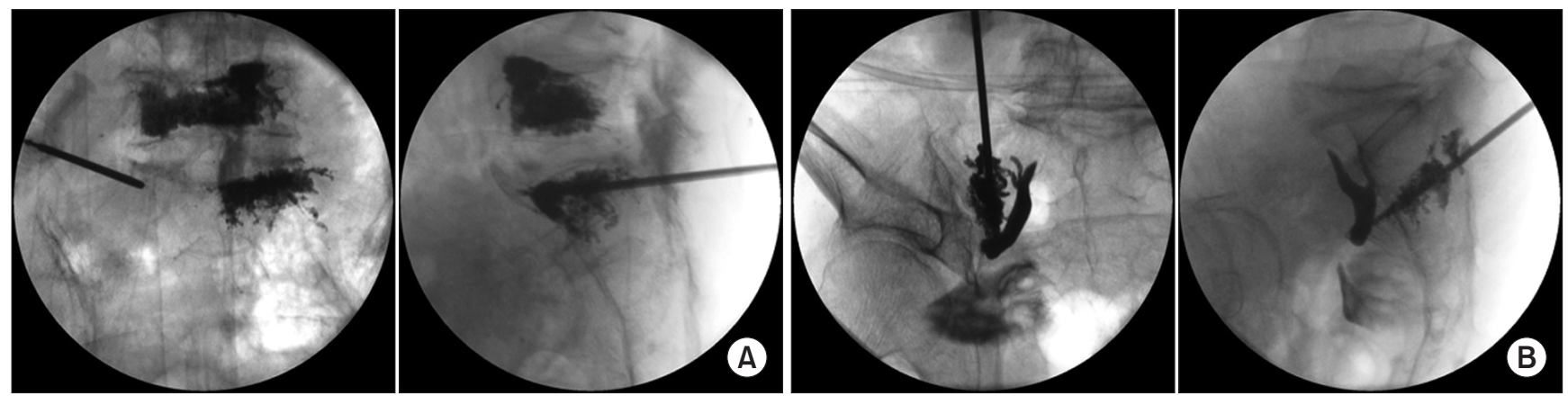

Fig. 6. Sacroplasty in the first sacral vertebral body. (A) Sacral body. The vertebral needle is inserted in the left S1 vertebral body in the anteroposterior fluoroscopic view. Bone cement is inserted from the anterior sacral vertebral body to the middle of the sacral vertebral body in the lateral fluoroscopic view. Percutaneous vertebroplasty at $L 5$ has already been done. (B) Sacral wing. The vertebral needle is inserted into the left sacral wing from the S1 to the S5, and then bone cement is inserted from the S5 to the S1 while withdrawing the needle in the anteroposterior and lateral fluoroscopic views, respectively. 
(from the cervical vertebrae to the coccyx) and the skull (temporal, sphenoid, ethmoid, zygomatic, maxilla, mandible, palatine, inferior nasal concha, and hyoid bone). Flat bones are commonly found in the human body, such as the skull (occipital, parietal, frontal, nasal, lacrimal, and vomer bone), the thoracic cage (sternum and ribs), and pelvis (ilium, ischium, and pubis), with the function of protecting their internal organs. Long bones include the humerus, radius, ulna, metacarpals, and phalanges in the upper extremities, and the femur, tibia, metatarsals, and phalanges in the lower extremities.

1) Bones in the thoracic cavity, scapula, and humerus

The origin of the cancers metastasized into the bones in the thoracic cavity and humerus are, commonly, the breast, thyroid glands, and lungs [3].

\section{(1) Costoplasty}

The ribs are the second most common metastatic bones from lung cancers, after the spine [34]. Most metastatic bone cases are found in both the thoracic spine and ribs.

The ribs can be divided into true ribs (R1-R7, anteriorly articulated with the sternum by the costal cartilages), false ribs (R8-R10, anteriorly articulated with the rib above), and floating ribs (R11 and R12, without articulation anteriorly) [35].

A rib is composed of the head, neck, and body. The head and neck of the first rib are attached to their own superior costal facet of the vertebral body and costal facet of the transverse process. From the 2nd to 10th ribs, the head of the rib is attached to both the rib above and their own vertebral bodies. The 11th and 12th ribs have their costal facets on the vertebral body alone, without the costal facets of the transverse process [36].

Patients with rib metastasis complain of pain, depending on their position. Pain is aggravated in the supine, lateral, or prone position when the rib metastasis involves the posterior, lateral, or anterior body of the rib. In addition to movement-related weight-bearing pain, incidental breakthrough pain can develop in coughing and sneezing.

Tenderness is apparent on multiple ribs or 2 or 3 points on the same ipsilateral rib. It is quite different from anterior or lateral chest pain from referred pain, originating from thoracic facet joint syndrome, which can be recognized by tenderness on the supra-facetal area. Corresponding lesion imaging, plain X-ray film, computed tomography/positron emission tomography-computed tomography, and bone scans, should match with palpation of the tenderness.

A $5 \mathrm{~cm}, 13$ gauge, short-bevel vertebral needle is suitable for costoplasty, in order not to pierce the pleura. The flat bone is composed of spongy bone between 2 compact bones. A perpendicular approach against bone osteolytic lesions is more convenient than approaching from above into the narrow upper margin or from below into the complicated complex of the intercostal costal nerve, artery,

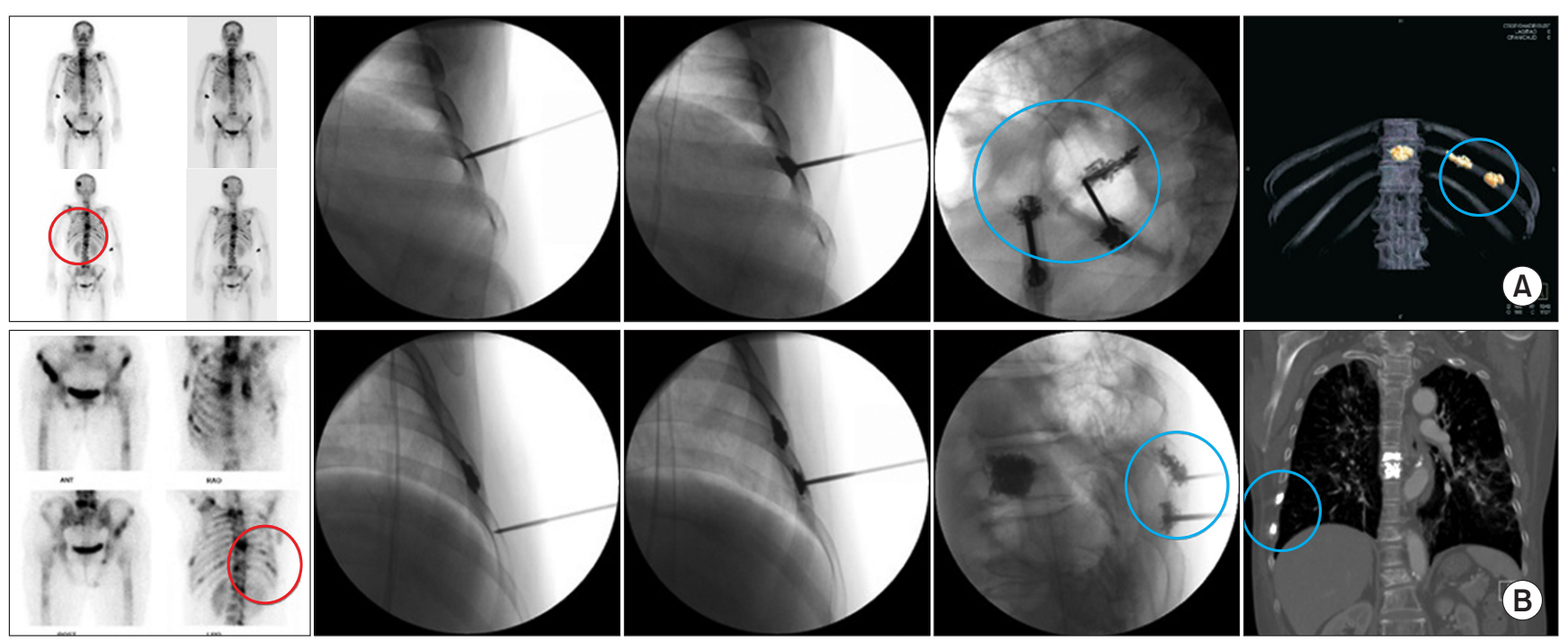

Fig. 7. Costoplasty. (A) A 66-year-old woman, diagnosed with lung cancer, shows active bone lesions in the skull, multiple vertebrae, and multiple ribs. Preoperative bone scans show multiple active bone lesions (the red circle), especially in the ribs. Intraoperative anteroposterior and lateral fluoroscopic views shows that two 13 gauge, $5 \mathrm{~cm}$ vertebral needles are placed in the left 10th rib. Postoperative computed tomography shows 2 apparent yellow bone cement shadows (the blue circle) in the body of the left 10th rib. Bone cement has already been inserted in the T10 vertebral body. (B) An 80 -yearold male, diagnosed with lung cancer, shows active bone lesions in multiple vertebrae and multiple ribs. He had already received percutaneous vertebroplasty at the T7 vertebral body. Bone cement was inserted twice at the different right 7th and 8th ribs. 
and vein. Anchoring to the slippery outer compact bone of the targeted rib requires hammering, while preventing penetration of the vertebral needle through the inner compact bone, using Kelly forceps. After the arrival of the vertebral needle inside the spongy bone, the stylet of the vertebral needle is removed and the cannula, without the stylet, should be fixed. Contrast medium should be injected for checking leakage into the vessels or pleura. Bone cement should be injected under lateral fluoroscopic monitoring. The amount of injected cement volume per each costoplasty is less than $2 \mathrm{~mL}$ (Fig. 7).

\section{(2) Sternoplasty}

The sternum is a blade-like flat bone, consisting of the manubrium, body and xiphoid process. The 1st costal notches of the manubrium articulate with the pair of the 1st costal cartilages. The 2nd costal notches at the sternal angle, between the manubrium and body, articulates with a pair of the 2nd costal cartilages. The body articulates with a pair of the 3rd to 7th costal cartilages at the 3rd to 7th costal notches. The true ribs, from the 1st to 7 th, articulate with the sternum.

Patients with sternal metastases complain of continuous pain or incidental pain during respiration. Tenderness should be proven to be osteolytic lesions in computed tomography or active lesions in bone scans. A 13 gauge, $5 \mathrm{~cm}$ vertebral needle is inserted perpendicular to the body of the sternum. The amount of injected cement volume per each sternoplasty is less than $2 \mathrm{~mL}$ (Fig. 8).

\section{(3) Scapuloplasty}

The scapula, or shoulder blade, is a triangular flat bone of the thoracic cage. It has 3 borders (medial [vertebral], lateral [axillary], and superior borders), and superior, inferior, and glenoid angles (or glenoid cavity). It also has costal and dorsal surfaces. When inserting the vertebral needle into the scapula, it is better to choose the medial and lateral borders, because the superior border contains the suprascapular notch, part of the route of the suprascapular nerve and artery. The suprascapular nerve originates from the ventral rami of the $\mathrm{C} 5$ and $\mathrm{C} 6$ spinal nerves. The suprascapular nerve innervates the supraspinatus and infraspinatus muscles, and receives sensory innervation from the acromioclavicular and glenohumeral joints.

Patients with scapula metastases complain of pain when they lie on their back, with/without persistent bone pain. Therefore, they usually sleep on their side. They sometimes have limited shoulder motion. Tenderness on the involved scapula is apparent. Imaging, including bone scans and positron emission tomography-computed tomography/computed tomography, should match the tenderness.

After drawing the 3 borders and scapular spine, tender points over the osteolytic lesions are also marked on the skin of the dorsal scapular surface. More than one vertebral needle may be needed if multiple osteolytic lesions exist. The superior border, especially the scapular notch, should be not be chosen for the entry of the vertebral needle, due to the passage of the suprascapular nerve and artery. An 11 gauge, $10 \mathrm{~cm}$ vertebral needle is required. After advancing the needle to the targeted lesion, a leakage test should be performed. Bone cement insertion is
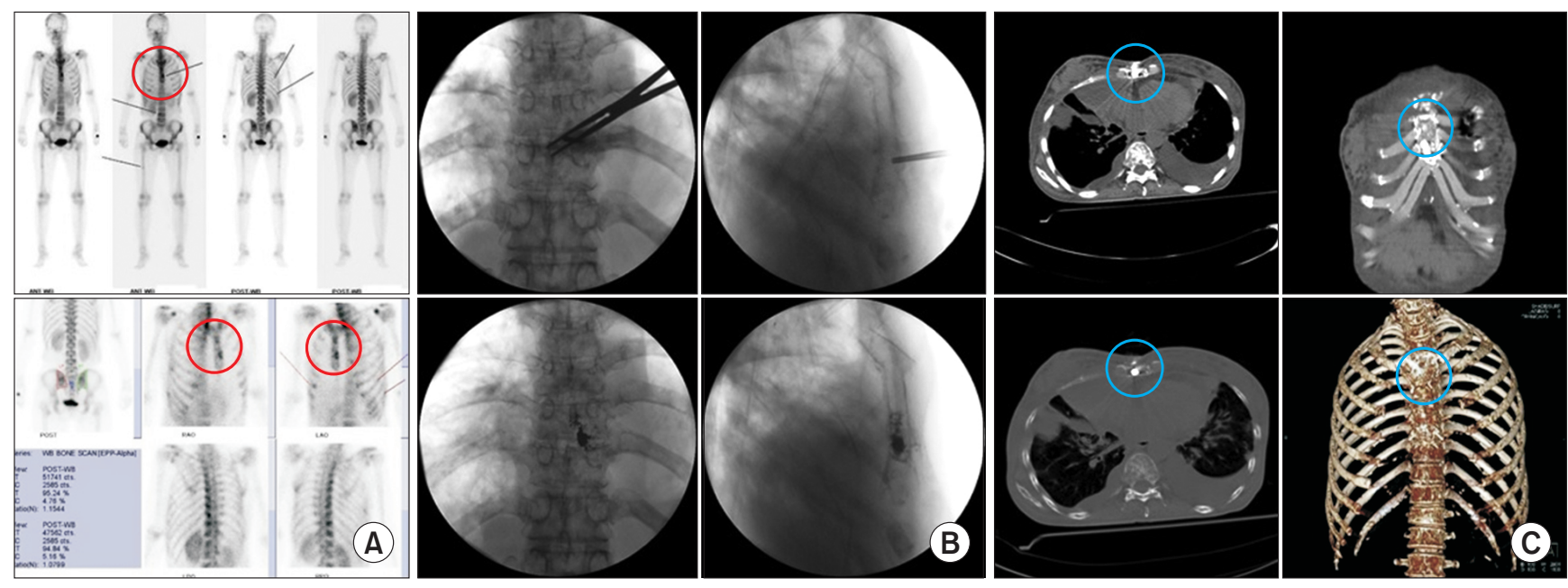

Fig. 8. Sternoplasty. A 49-year-old woman, diagnosed with breast cancer, complained of pain in breathing. (A) Preoperative bone scans. Tenderness on the mid-sternal body matches an active bone lesion (the red circle). (B) Intraoperative fluoroscopic views. An 11 gauge, $5 \mathrm{~cm}$ vertebral needle is inserted into the mid-sternal body. Kelly forceps are used for preventing the vertebral needle piercing the inner surface of the sternal body. Bone cement is inserted under lateral fluoroscopic monitoring. (C) Postoperative computed tomography. The inserted bone cement (the blue circle) is apparent in the sternum in axial computed tomography. 
performed while withdrawing the needle (Fig. 9) [37,38]. The injected volume of the cement is variable according to osteolytic lesions. Pain relief can be immediate after POP in the scapula, when lying on the back or in a dependent position.

\section{(4) Humeroplasty}

The humerus is a long bone. It consists of 2 epiphyses (proximal end [head and anatomical/surgical neck] and distal end) and 1 diaphysis (shaft). POP, using bone cement, is effective for a metastasis to the proximal end rather than the shaft, which has an intramedullary canal.

The greater tubercle is the common insertion site of the supraspinatus, infraspinatus, and teres minor muscles of the rotator cuff. The lesser tubercle is the insertion site of the subscapular muscle of the rotator cuff. The bicipital groove is the passage of the long head of the biceps brachii. The deltoid and pectoral branches of the thoracoacromial artery and anterior and posterior circumflex humeral arteries supply blood to the humeral head and neck. Therefore, the greater tubercle, lesser tubercle, bicipital groove, as well as the thoracoacromial and circumflex humeral arteries should be avoided during entry of the vertebral needle.

Patients with humeral head metastases may have pain and limited shoulder motion. Tenderness on the humeral head should match imaging, including computed tomography/magnetic resonance imaging and bone scans. Vertebral needle insertion, a leakage test, and bone cement insertion are performed under fluoroscopy, and proper cement insertion is confirmed by postoperative computed tomography (Fig. 10). The amount of injected bone cement is less than $10 \mathrm{~mL}$.

\section{2) Pelvic bones and femur}

The pelvic girdle is composed of the 2 coxal bones and the sacrum. The 2 coxal bones are connected with each other at the cartilaginous symphysis pubis, and to the sacrum via the sacroiliac joints. The orientation of the ilium, ischium, and pubis of the coxal bones is quite easy to understand from the tri-radiate cartilage of a child's acetabulum: (1) the ilium: superiorly, (2) the ischium: inferiorlyposteriorly, and (3) the pubis: inferiorly-anteriorly [39].

Metastases to the pelvic bones and femoral head commonly originate from prostate cancer, renal cancer, colorectal cancer, as well as breast and thyroid cancer $[40,41]$.
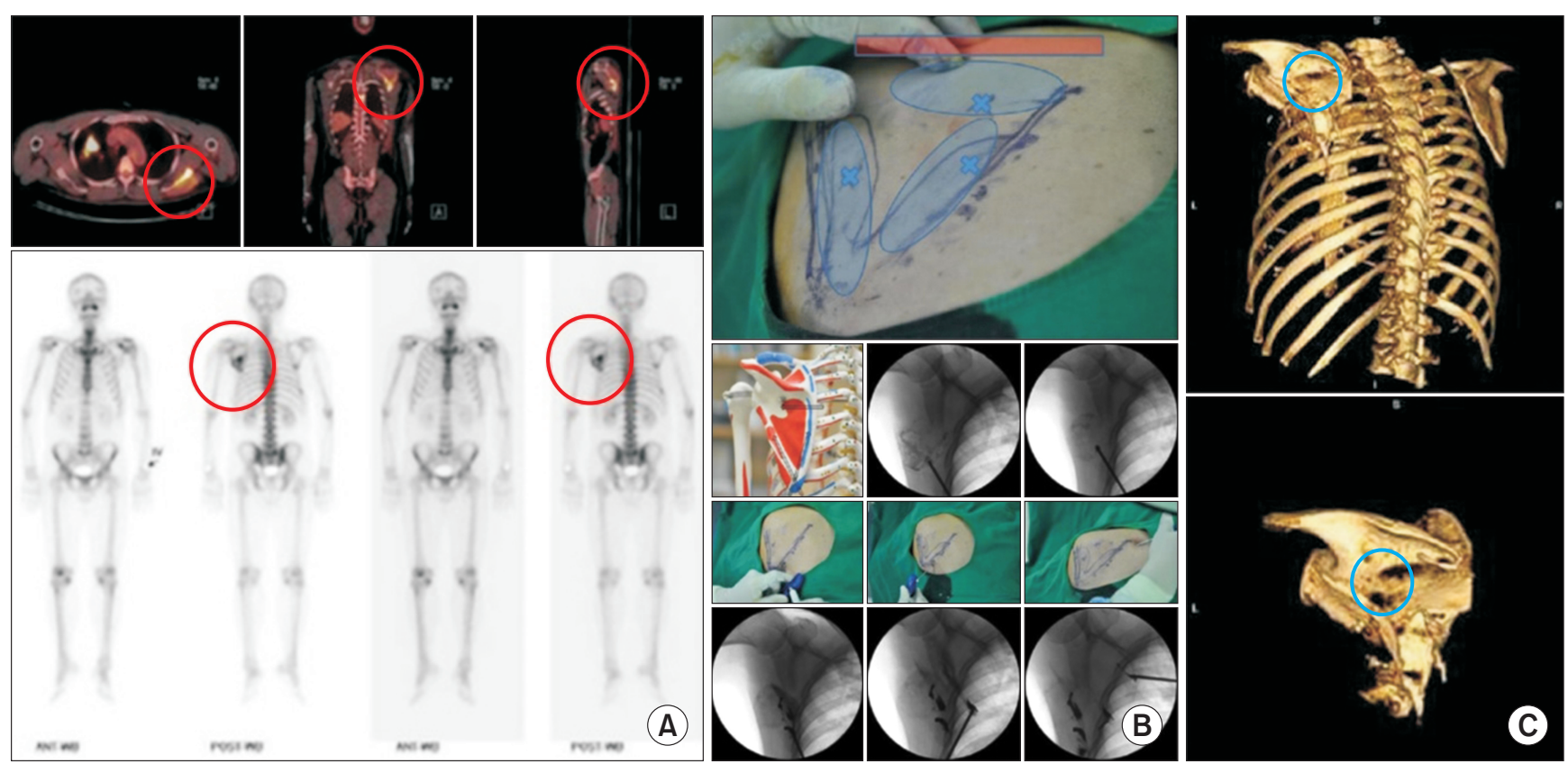

Fig. 9. Scapuloplasty. A 59-year-old man, diagnosed with lung cancer, complained of pain when he lay on his back and in a left lateral decubitus position. (A) Positron emission tomography-computed tomography shows a bright yellow lesion (the red circle) in the left scapula in axial, coronal, and sagittal sections. Bone scans also shows an active lesion on the left scapula (the red circle). Tender points matched these images. (B) Intraoperative images. Three tender points (the blue Xs), the medial and lateral borders, and scapular spine (the red bar) are drawn over the left scapula. Three vertebral needles are inserted from the inferior angle to the lateral and medial borders, and from the medial border to the middle of the scapula. (C) Postoperative computed tomography. Bone cement (the blue circle) is inserted after confirmation of no leakage using contrast medium. Adapted from the article of Choi et al. (Pain Physician 2010; 13: 485-91) [37]. 

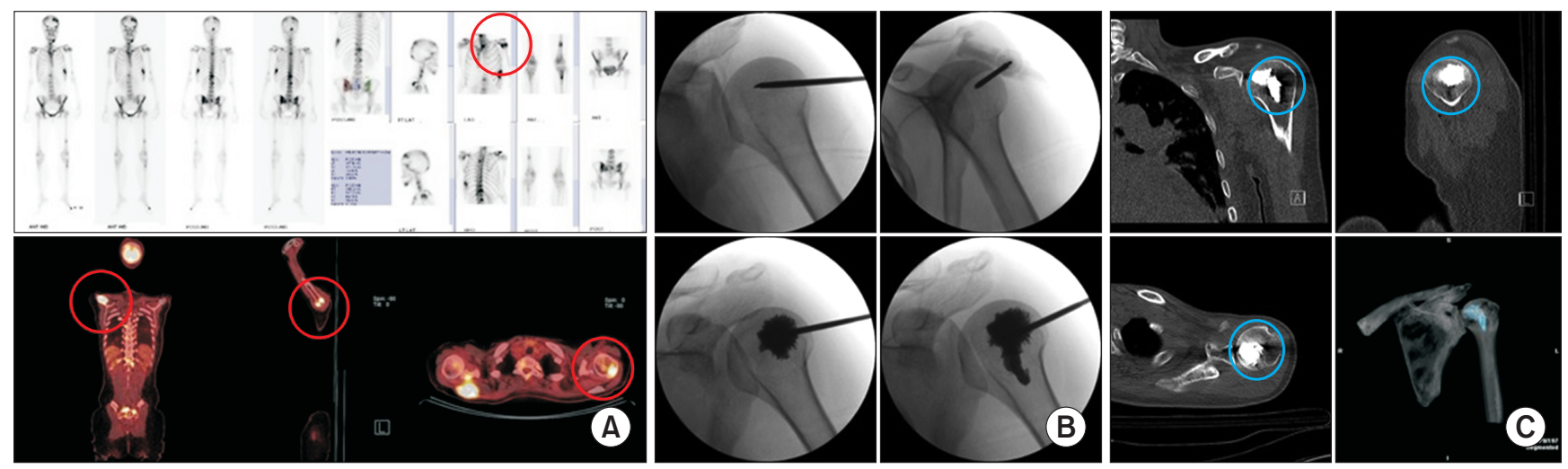

Fig. 10. Humeroplasty. A 40-year-old woman, diagnosed with breast cancer, complained of left shoulder pain and limitation of motion. Tenderness on the left humeral head was noted. (A) Bone scans and positron emission tomography-computed tomography shows a lesion in the left humeral head (the red circle). (B) Bone cement is inserted through the 11 gauge, $10 \mathrm{~cm}$ vertebral needle in fluoroscopic views. (C) Postoperative computed tomography shows cement filling (the blue circle) in the left humeral head.
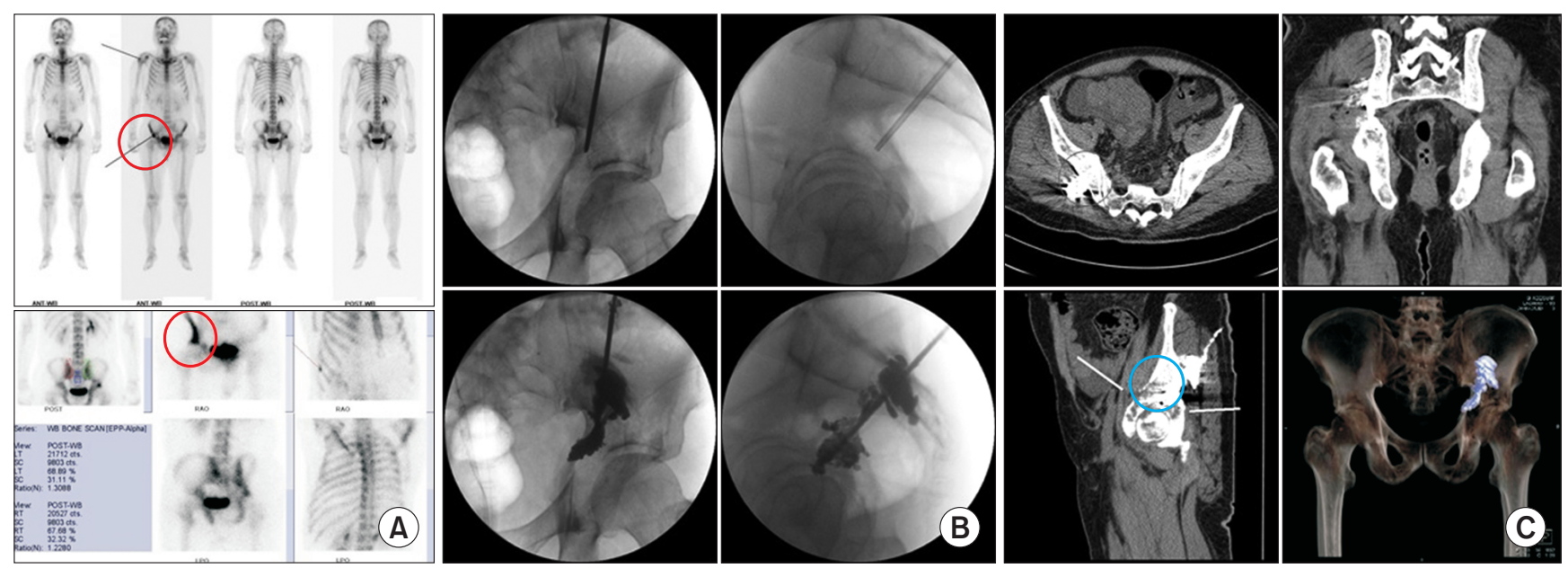

Fig. 11. Ilioplasty. A 61-year-old male, diagnosed with hepatocellular carcinoma, complained of right hip pain in a weight-bearing position. Tenderness was noted on the right ilium near the hip joint. (A) Preoperative bone scans show an active lesion (the red circle) in the right ilium. (B) A vertebral needle is inserted from the right iliac crest through the iliac wing, through the iliac body, and into the superior acetabulum in the anteroposterior and lateral fluoroscopic views. Bone cement is inserted from the right acetabulum to the iliac body in the anteroposterior and lateral fluoroscopic views. (C) Postoperative computed tomography shows injected bone cement (the blue circle) in the osteolytic lesion in the superior acetabulum and iliac body.

\section{(1) Ilioplasty}

The iliac crest between the anterior superior iliac spine and posterior superior iliac spine is the most common site for bone marrow biopsy. The entry point of the vertebral needle for POP of the ilium is the iliac crest, just as with bone marrow biopsy. The needle should be advanced along with the curvature of the ilium from the iliac crest to the superior acetabulum, if necessary. Caution should be given not to insert bone cement into the sacroiliac joint (Fig. 11). The injected cement volume per each ilioplasty is less than $10 \mathrm{~mL}$.
(2) Ischioplasty

The ischium consists of the body, superior ramus, and inferior ramus. The entry point of the vertebral needle for ischioplasty is the ischial tuberosity, in a prone position. If necessary, the needle should be advanced into the inferoposterior acetabulum (Fig. 12). The injected cement volume per each ischioplasty is less than $5 \mathrm{~mL}$.

\section{(3) Puboplasty}

The pubic bone is located inferiorly and anteriorly among the pelvic bones. It consists of a body, superior ramus, and inferior ramus. The left and right bodies of the pubic bones join together at the symphysis pubis. An 11 gauge, 5 

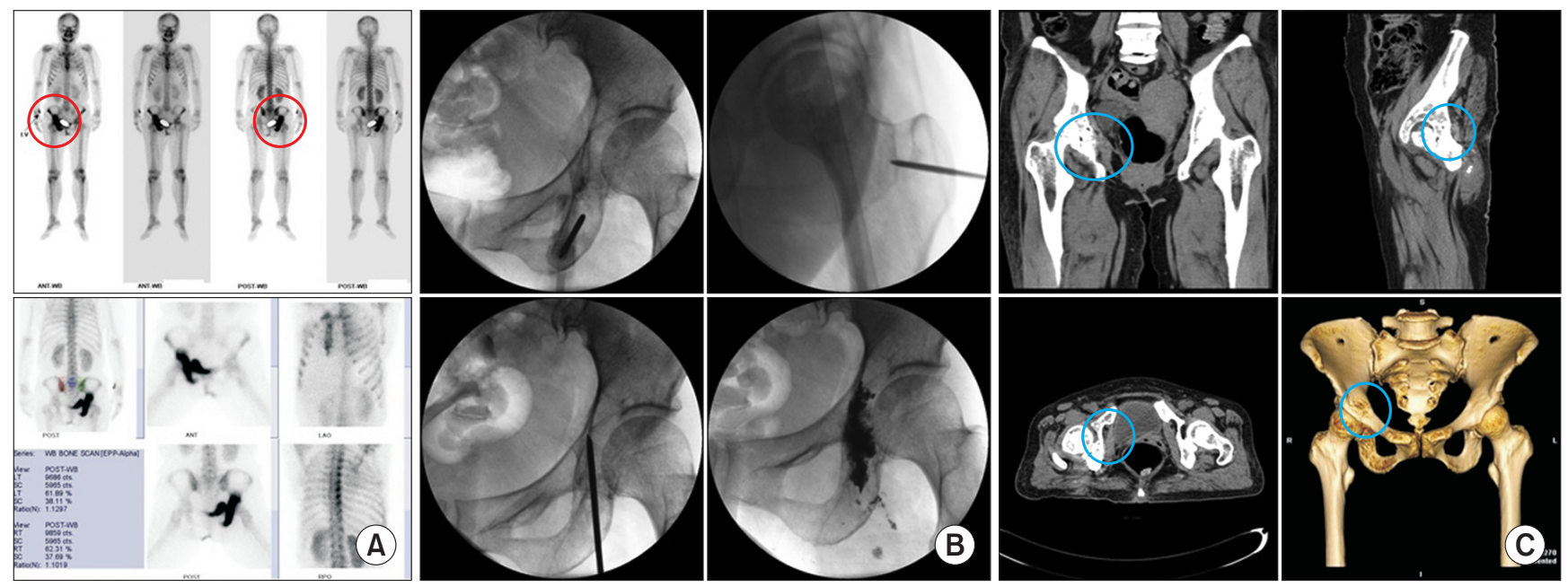

Fig. 12. Ischioplasty. A 47-year-old woman, diagnosed with adenocarcinoma of the lung, complained of right hip pain in a weight-bearing position. Tenderness was noted on the right ischium near the hip joint. (A) Preoperative bone scans show an active lesion (the red circle) on the right ischial body and inferoposterior acetabulum. (B) Intraoperative fluoroscopic views show that an 11 gauge, $10 \mathrm{~cm}$ vertebral needle is inserted from the right ischial tuberosity to the ischial body and inferoposterior acetabulum. (C) Postoperative computed tomography shows cement filling (the blue circle) in the right ischium.
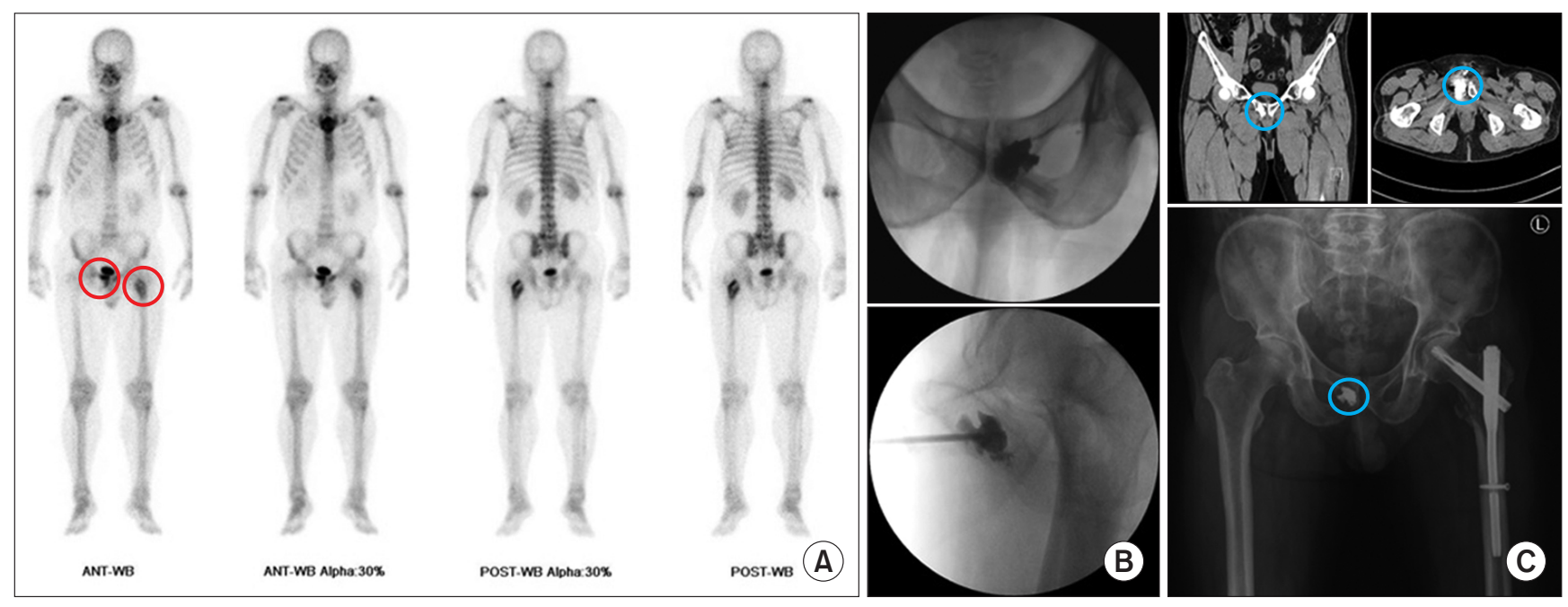

Fig. 13. Puboplasty. A 65-year-old man, diagnosed with hepatocellular carcinoma, complained of continuous pain in the pubis. Tenderness was more prominent on the right pubis and left femur. (A) Preoperative bone scans show active lesions (the red circle) on the right pubis and left femur. (B) An 11 gauge, $10 \mathrm{~cm}$ vertebral needle is inserted from the right pubic tubercle. (C) Postoperative computed tomography shows cement filling (the blue circle) in the right pubis. After puboplasty, intramedullary nail fixation, as a prophylactic surgery for intertrochanteric fracture in the left femur, was performed by an orthopedic surgeon.

$\mathrm{cm}$ vertebral needle is inserted perpendicular to the pubic tubercle (Fig. 13). The injected cement volume per each puboplasty is less than $5 \mathrm{~mL}$.

\section{(4) Femoroplasty}

Unlikely POP at the humeral head, POP at the femoral head alone without intramedullary fixation of the femoral shaft is unstable, because the femur and acetabulum of the pelvic bones are weight-bearing units. In case of greater or lesser trochanter metastases, after intramedullary fixation of the femoral shaft, POP at the greater or lesser trochanter may be needed (Fig. 14). The injected cement volume per each femoral head osteoplasty is less than $10 \mathrm{~mL}$.

\section{Extraspinal POP in OCLs (osteochondritis dissecans)}

An OCL is an injury or small fracture of the cartilage surface and subchondral bone, commonly in the talus or tibia 

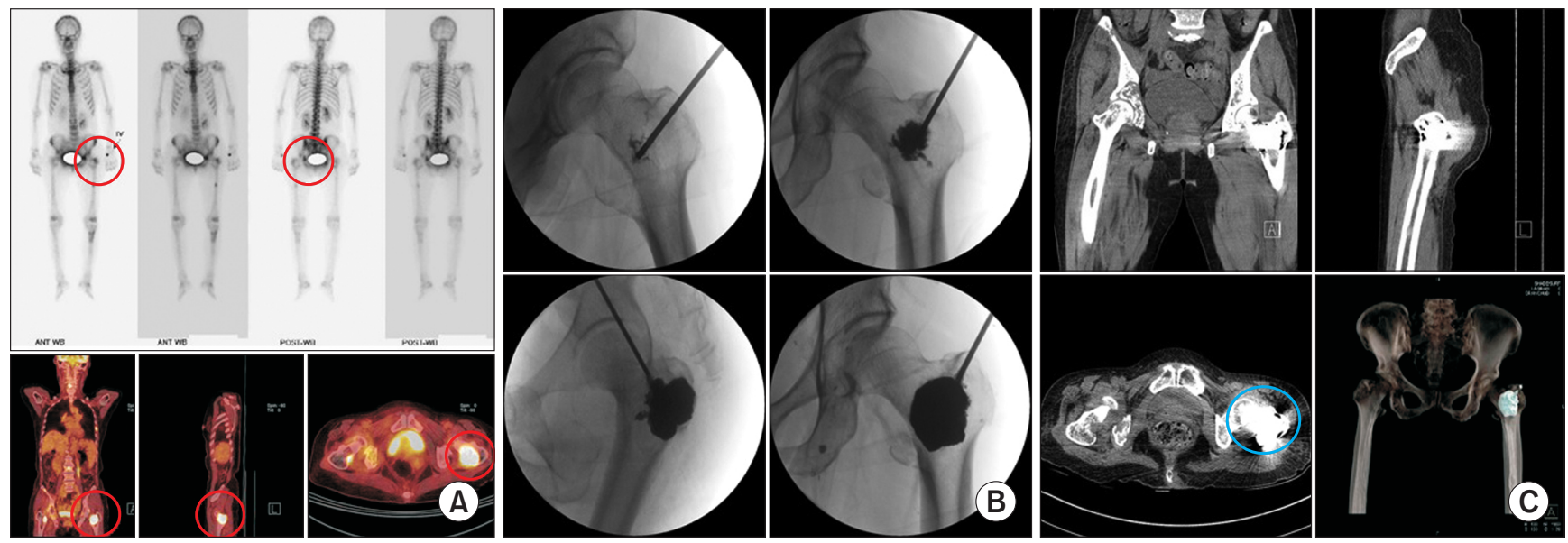

Fig. 14. Femoroplasty. A 73-year-old woman, diagnosed with hepatocellular carcinoma, complained of left hip pain. (A) Preoperative bone scans and positron emission tomography-computed tomography show an active lesion (the red circle) on the left femoral head. (B) An 11 gauge, $10 \mathrm{~cm}$ vertebral needle is inserted from the greater trochanter into the femoral head along the intertrochanteric line. (C) Postoperative computed tomography shows cement (the blue circle) filling in the left femoral neck.
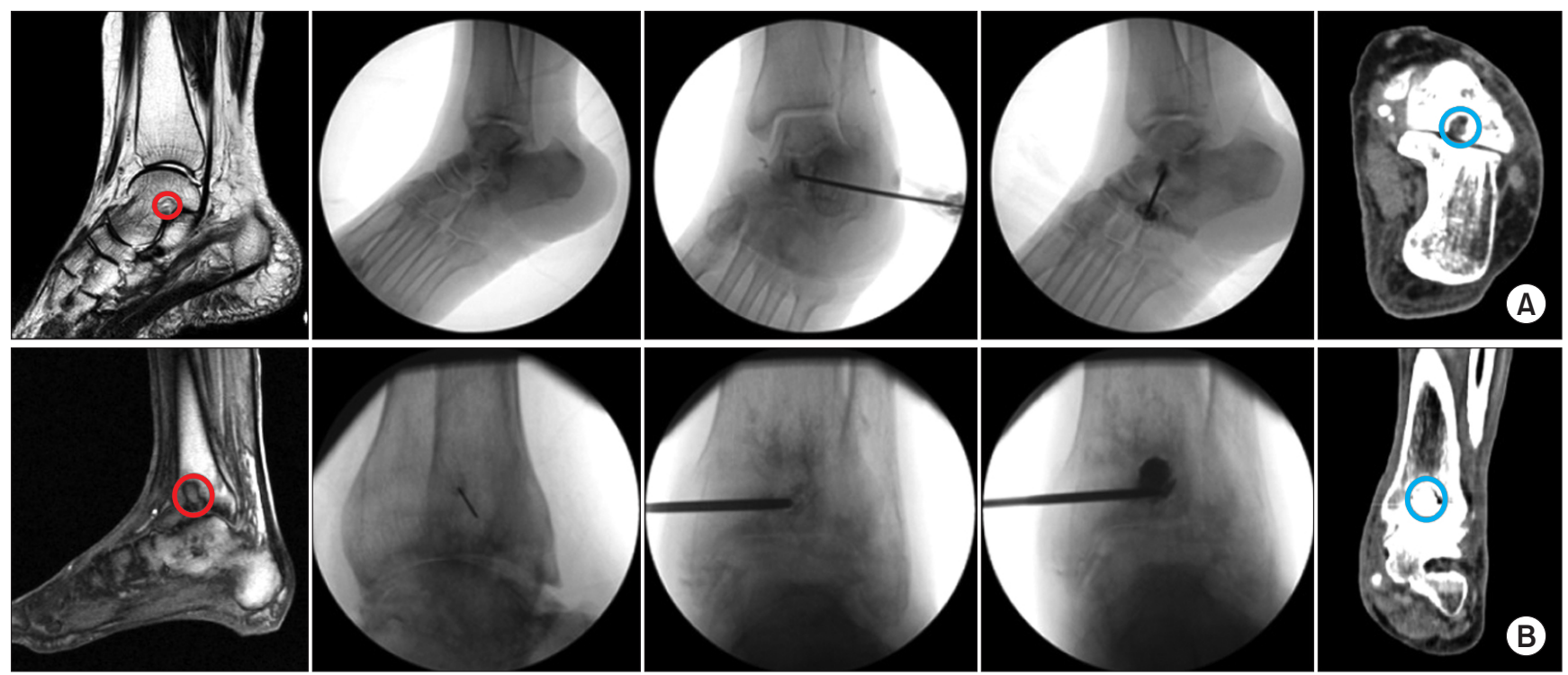

Fig. 15. Percutaneous osteoplasty (POP) for osteochondral lesions (OCLs) of the talus and tibia using calcium phosphate or hydroxyapatite bone cement. (A) POP at the OCL of the talus. Preoperative magnetic resonance imaging (MRI) shows an osteochondral lesion (the red circle) of the talus. Intraoperative fluoroscopic view shows insertion of a vertebral needle and bone cement (calcium phosphate or hydroxyapatite) after confirming no leakage into the joint or blood vessels. Postoperative computed tomography (CT) shows properly placed bone cement (the blue circle) in the OCLs of the talus. (B) POP at the OCL of the talus. Preoperative MRI shows an OCL of the tibia. After local infiltration, a 11 gauge, $10 \mathrm{~cm}$ vertebral needle is inserted. After confirmation of no leakage into the vessel, bone cement is injected. Postoperative CT shows proper cement (the blue circle) filling in the tibia. Adapted from the article of Ri et al. (Korean J Pain 2013; 26: 164-8) [10].

$[10,42]$. The talus articulates with the tibia and fibula superiorly, forming a mortise, with the navicular anteriorly, and calcaneus inferiorly.

Instead of using PMMA cement in metastatic bone lesions, calcium phosphate or HAC is used in order to reduce thermal ablation and increase osteoconduction. Monitoring under arthroscopy and fluoroscopy decreases the risk of cement leakage into the ankle joint (Figs. 15, 16). The injected cement volume for OCL of talus or tibia is less than 2 or $5 \mathrm{~mL}$, respectively.

\section{CONCLUSIONS}

For the decision regarding and evaluation of POPs, the preoperative revised Tokuhashi scoring system for the evaluation of patient life expectancy with bone metastases, preoperative and postoperative comparison of KPS 

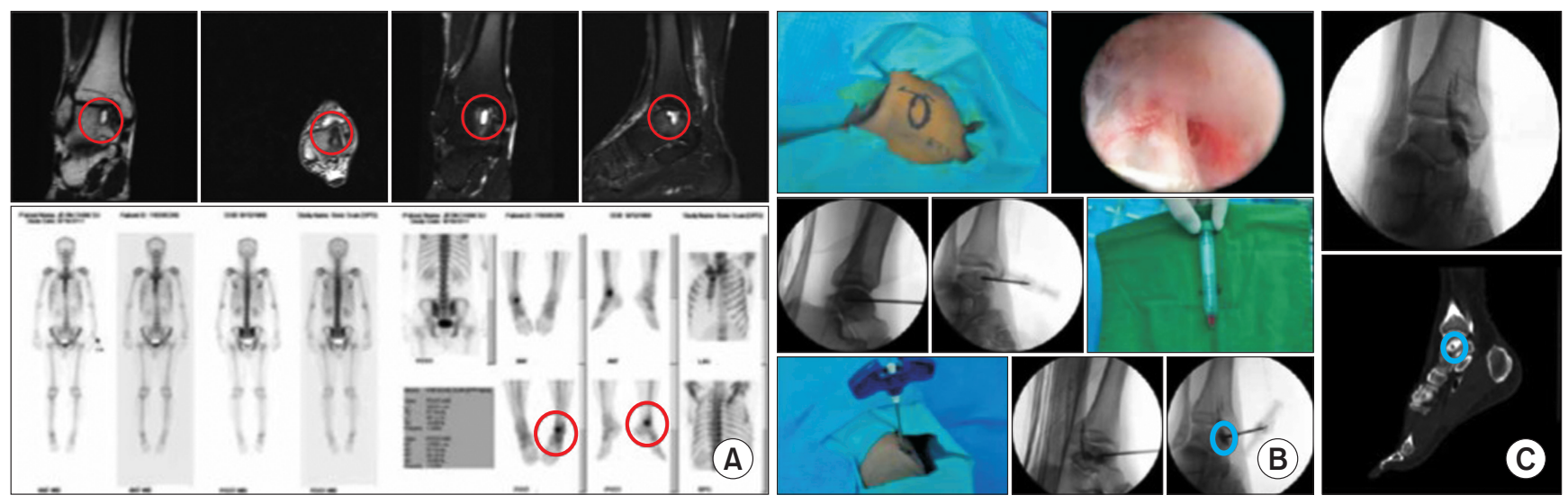

Fig. 16. Percutaneous osteoplasty (POP) for osteochondral lesion (OCL) of the talus under arthroscopic monitoring. (A) Preoperative magnetic resonance imaging and bone scans reveal an OCL (the red circle) of the talus. (B) Intraoperative POP using calcium phosphate or hydroxyapatite bone cement after aspiration of joint fluid under fluoroscopy and arthroscopy. (C) Postoperative plain X-ray and computed tomography show inserted bone cement (the blue circle). Adapted from the article of Seo et al. (Pain Physician 2012; 15: E743-8) [42].

and ECOG PS for comparing the effectiveness of therapies and accessing the prognosis of patients, and the preoperative Mirels score, for investigating the risk of pathologic fracture, are also required.

It should be emphasized that adjacent facet or shoulder/ sacroiliac joint/hip joint injections are essential prior to POPs. They help to determine whether to perform POPs after removal of adjacent joint pain, to permit patients be more cooperative in assuming specific operating positions, and in determining the precise current levels of pain among mixed old/new and painful/non-painful multiple vertebral fractures.

For reducing the risk of leakage into the blood vessels or epidural space, augmentation by injecting cement volume or recovery of original bone shape after POP is less important because other mechanisms for pain relief by POP, such as chemical or thermal ablation of the nociceptive nerves, exist.

In conclusion, even though POP is a simple and fast pain relief method for painful bony lesions, basic understanding of anatomy, related to the shape of the various bones and adjacent blood vessels and nerves, is required. Decreased analgesic requirements and increased physical performance will be shown immediately after removal of deep somatic pain by POP. POPs are helpful for both painful malignant osteolytic/osteoblastic metastases and benign osteoporotic vertebral compression fracture/OCLs.

\section{CONFLICT OF INTEREST}

No potential conflict of relevant to this article was reported.

\section{FUNDING}

This study was supported by a research grant from Pusan National University Yangsan Hospital in 2021.

\section{ORCID}

Won-Sung Kim, https://orcid.org/0000-0002-8905-9521

Kyung-Hoon Kim, https://orcid.org/0000-0003-3925-8917

\section{REFERNCES}

1. Deramond H, Depriester C, Galibert P, Le Gars D. Percutaneous vertebroplasty with polymethylmethacrylate. Technique, indications, and results. Radiol Clin North Am 1998; 36: 533-46.

2. Schuenke M, Schulte E, Schumacher U. Atlas of anatomy: general anatomy and musculosketel system. New York, Thieme. 2006, p 34.

3. Macedo F, Ladeira K, Pinho F, Saraiva N, Bonito N, Pinto L, et al. Bone metastases: an overview. Oncol Rev 2017; 11: 321.

4. Singh V, Taunk A, Phadke RV, Neyaz Z, Prasad SN. Analysis of percutaneous vertebroplasty-a prospective study. Egypt J Radiol Nucl Med 2019; 50: 21.

5. Vaishya R, Chauhan M, Vaish A. Bone cement. J Clin Orthop Trauma 2013; 4: 157-63.

6. Yousefi AM. A review of calcium phosphate cements and acrylic bone cements as injectable materials for bone repair and implant fixation. J Appl Biomater Funct Mater 2019; 17: 2280800019872594.

7. Jackson IT, Yavuzer R. Hydroxyapatite cement: an alternative for craniofacial skeletal contour refinements. Br J Plast 
Surg 2000; 53: 24-9.

8. Nicholson JW. Maturation processes in glass-ionomer dental cements. Acta Biomater Odontol Scand 2018; 4: 63-71.

9. Giannoudis PV, Dinopoulos H, Tsiridis E. Bone substitutes: an update. Injury 2005; 36 Suppl 3: S20-7.

10. Ri HS, Lee DH, Kim KH. Searching for hidden, painful osteochondral lesions of the ankle in patients with chronic lower limb pain - two case reports -. Korean J Pain 2013; 26: 164-8.

11. Kim TK, Kim KH, Kim CH, Shin SW, Kwon JY, Kim HK, et al. Percutaneous vertebroplasty and facet joint block. J Korean Med Sci 2005; 20: 1023-8.

12. Yoon JY, Kim TK, Kim KH. Anterolateral percutaneous vertebroplasty at $\mathrm{C} 2$ for lung cancer metastasis and upper cervical facet joint block. Clin J Pain 2008; 24: 641-6.

13. Mirels H. Metastatic disease in long bones. A proposed scoring system for diagnosing impending pathologic fractures. Clin Orthop Relat Res 1989; 249: 256-64.

14. Tokuhashi Y, Matsuzaki H, Oda H, Oshima M, Ryu J. A revised scoring system for preoperative evaluation of metastatic spine tumor prognosis. Spine (Phila Pa 1976) 2005; 30: 2186-91.

15. Péus D, Newcomb N, Hofer S. Appraisal of the Karnofsky Performance Status and proposal of a simple algorithmic system for its evaluation. BMC Med Inform Decis Mak 2013; 13: 72 .

16. Verger E, Salamero M, Conill C. Can Karnofsky performance status be transformed to the Eastern Cooperative Oncology Group scoring scale and vice versa? Eur J Cancer 1992; 28A: 1328-30.

17. Gilroy AM, MacPherson BR. Atlas of anatomy. 3rd ed. New York, Thieme. 2016, p 9.

18. Kavakebi P, Girod PP, Hartmann S, Tschugg A, Thomé C. Transoral vertebroplasty of the lateral mass of $\mathrm{Cl}$ using image guidance. Acta Neurochir (Wien) 2017; 159: 1159-62.

19. Gilroy AM, MacPherson BR. Atlas of anatomy. 3rd ed. New York, Thieme. 2016, p 17.

20. Seo SS, Lee DH, Kim HJ, Yoon JW, Kwon OS, Kim KH. Percutaneous vertebroplasty at $\mathrm{C} 7$ for the treatment of painful metastases -a case report-. Korean J Anesthesiol 2013; 64: 276-9.

21. Park SC, Kim KH. Effect of adding cervical facet joint injections in a multimodal treatment program for long-standing cervical myofascial pain syndrome with referral pain patterns of cervical facet joint syndrome. J Anesth 2012; 26: 73845 .

22. Kim KH, Choi SH, Kim TK, Shin SW, Kim CH, Kim JI. Cervical facet joint injections in the neck and shoulder pain. J Korean Med Sci 2005; 20: 659-62.

23. Kim KH, Kim TK. Unipedicular approach for percutaneous vertebroplasty. In: Minimally invasive percutaneous spinal techniques. Edited by Kim DH, Kim YC, Kim KH. Philadelphia, Elsevier Saunders. 2010, pp 270-6.

24. Lee JH, Jeon GR, Ro JH, Byoen GJ, Kim TK, Kim KH. Evalu- ation of an experimentally designed stereotactic guidance system for determining needle entry point during uniplanar fluoroscopy-guided intervention. Korean J Pain 2012; 25: 818.

25. Gilroy AM, MacPherson BR. Atlas of anatomy. 3rd ed. New York, Thieme. 2016, pp 36, 68, 184.

26. Shin HJ, Choi YM, Kim HJ, Lee SJ, Yoon SH, Kim KH. Retroperitoneal hemorrhage from an unrecognized puncture of the lumbar right segmental artery during lumbar chemical sympathectomy: diagnosis and management. J Clin Anesth 2014; 26: 671-5.

27. Shimizu S, Tanaka R, Kan S, Suzuki S, Kurata A, Fujii K. Origins of the segmental arteries in the aorta: an anatomic study for selective catheterization with spinal arteriography. AJNR Am J Neuroradiol 2005; 26: 922-8.

28. Santillan A, Nacarino V, Greenberg E, Riina HA, Gobin YP, Patsalides A. Vascular anatomy of the spinal cord. J Neurointerv Surg 2012; 4: 67-74.

29. Joo YC, Park JY, Kim KH. Comparison of alcohol ablation with repeated thermal radiofrequency ablation in medial branch neurotomy for the treatment of recurrent thoracolumbar facet joint pain. J Anesth 2013; 27: 390-5.

30. Maccauro G, Spinelli MS, Mauro S, Perisano C, Graci C, Rosa MA. Physiopathology of spine metastasis. Int J Surg Oncol 2011; 2011: 107969.

31. Araujo JL, Veiga JC, Figueiredo EG, Barboza VR, Daniel JW, Panagopoulos AT. Management of metastatic spinal column neoplasms--an update. Rev Col Bras Cir 2013; 40: 508-14.

32. Alexandru D, So W. Evaluation and management of vertebral compression fractures. Perm J 2012; 16: 46-51.

33. Fujii M, Abe K, Hayashi K, Kosuda S, Yano F, Watanabe S, et al. Honda sign and variants in patients suspected of having a sacral insufficiency fracture. Clin Nucl Med 2005; 30: 165-9.

34. Chen YQ, Yang Y, Xing YF, Jiang S, Sun XW. Detection of rib metastases in patients with lung cancer: a comparative study of MRI, CT and bone scintigraphy. PLoS One 2012; 7: e52213.

35. Gilroy AM, MacPherson BR, Wikenheiser JC. Atlas of anatomy. 4th ed. New York, Thieme. 2020, p 51.

36. Gilroy AM, MacPherson BR, Wikenheiser JC. Atlas of anatomy. 4th ed. New York, Thieme. 2020, p 10.

37. Choi HR, Lee PB, Kim KH. Scapuloplasty alleviates scapular pain resulting from lung cancer metastasis. Pain Physician 2010; 13: 485-91.

38. Lee JH, Kim SY, Ok HG, Kim TK, Kim KH. Extraspinal percutaneous osteoplasty for the treatment of painful bony metastasis. J Korean Med Sci 2018; 33: e61.

39. Gilroy AM, MacPherson BR, Wikenheiser JC. Atlas of anatomy. 4th ed. New York, Thieme. 2020, pp 230-1.

40. Khattak MJ, Ashraf U, Nawaz Z, Noordin S, Umer M. Surgical management of metastatic lesions of proximal femur and the hip. Ann Med Surg (Lond) 2018; 36: 90-5.

41. Müller DA, Capanna R. The surgical treatment of pelvic bone 
metastases. Adv Orthop 2015; 2015: 525363.

42. Seo SS, Park JY, Kim HJ, Yoon JW, Park SH, Kim KH. Percutaneous osteoplasty for the treatment of a painful osteochon- dral lesion of the talus: a case report and literature review. Pain Physician 2012; 15: E743-8. 